\title{
Escenarios de mitigación de \\ emisiones para el sector rural del Estado de Chiapas utilizando modelos de estados y transiciones
}

\section{Emission mitigation scenarios for the rural sector of the State of Chiapas using state models and transitions}

\author{
Sara Covaleda', Fernando Paz-Pellat ${ }^{1,2^{*}}$ y Alejandro Ranero'
}

\begin{abstract}
1 Kibeltik Clima y Medio Ambiente A. C. San Cristóbal de las Casas, Chiapas.

2 GRENASER - Colegio de Postgraduados Campus * *Autor de correspondencia. ferpazpel@gmail.com Montecillo. Montecillo, Estado de México.
\end{abstract}

\section{RESUMEN}

En el estado de Chiapas, donde las emisiones de los sectores Uso del Suelo, Cambio de Uso del Suelo y Silvicultura [Uscusys] y Agrícola (incluida la ganadería) abarcan $78 \%$ de las emisiones totales, es necesario plantear actividades de mitigación en el sector rural (en particular, la reducción de emisiones por deforestación y degradación forestal). En este trabajo se generaron curvas de costos de abatimiento y escenarios de mitigación para Chiapas utilizando los modelos de estados y transiciones (METs) elaborados para ocho regiones del estado. Estos modelos permiten conocer los impactos asociados con la transición entre un estado (uso del suelo o tipo de vegetación) inicial y otro final en términos de carbono y factores socio-económicos. Las restricciones consideradas fueron: costos de oportunidad positivos y negativos, subsidio, crédito y tasa de interés. El estado presenta un gran potencial de mitigación de emisiones en el sector rural, en particular las regiones Selva Maya y Altos. El análisis de distintos escenarios muestra que un costo de oportunidad de USD 4/t CO a USD 6/t $\mathrm{CO}_{2}$ es suficiente para una buena porción del potencial de mitigación de gases de efecto invernadero de Chiapas. El contar con subsidio y crédito para las actividades de implementación afecta el potencial de mitigación para determinados intervalos de costos de oportunidad; asimismo, las actividades con costos de oportunidad negativos son un área de oportunidad para mitigar emisiones reorientando las políticas públicas. Para mejorar la capacidad de análisis de los modelos es necesario incentivar la investigación sobre actividades relacionadas con la degradación forestal, manejo forestal sostenible e incremento de almacenes de carbono forestales y agropecuarios.

PAlAbRAs ClAve: costos de oportunidad, curvas de abatimiento unitarias, REDD+, sector agropecuario.

\section{ABSTRACT}

In the State of Chiapas, where emissions from the LULUCF (Land Use, Land Use Change and Forestry) and Agricultural (including livestock) sectors cover $78 \%$ of total emissions, it is necessary to propose mitigation activities in the rural sector (in particular the reduction of emissions from deforestation and forest degradation). In this work, abatement cost curves and mitigation scenarios for Chiapas were generated using state and transition models prepared for eight regions of the state. These models allow us to know the impacts associated with the transition between an initial state (land use or vegetation type) and a final one in terms of carbon and socio-economic factors. The restrictions considered were: positive and negative opportunity costs, subsidy, credit and interest rate. The state has great potential to mitigate emissions in the rural sector, particularly the Selva Maya and Altos regions. The analysis of different scenarios shows that an opportunity cost for USD 4/t $\mathrm{CO}_{2}-$ USD 6/t $\mathrm{CO}_{2}$ is enough for a good portion of the GHG mitigation potential of Chiapas. The subsidy and credit for the implementation activities affects the mitigation potential for certain ranges of opportunity costs, also, the activities with negative opportunity costs are an opportunity area to mitigate emissions reorienting public policies. To improve the analysis capacity of the models it is necessary to encourage research on activities related to forest degradation, sustainable forest management and increase of forest and agricultural carbon stores.

KEYWORDS: opportunity costs, abatement curves, REDD+, agricultural sector. 


\section{INTRODUCCIÓN}

Chiapas es un estado comprometido con la lucha contra el cambio climático; por ello, desde el año 2009 las instituciones gubernamentales apoyadas por centros de investigación y organizaciones de la sociedad civil han realizado grandes esfuerzos para preparar a la entidad para echar a andar los nuevos mecanismos de mitigación de emisiones discutidos en la Convención Marco de Naciones Unidas sobre el Cambio Climático [Cmnucc], en particular el mecanismo REDD+ (Reducción de emisiones por deforestación y degradación forestal más conservación, incrementos en los almacenes de carbono y manejo forestal sustentable). Este mecanismo es contemplado en el reciente Acuerdo de París de la Cmnucc, que en su Artículo 5 reconoce el papel de los bosques como sumideros y reservorios de carbono y se alienta a las partes a adoptar enfoques de política e incentivos positivos para REDD+, mediante pagos basados en resultados (Cmnucc, 2015).

El Programa de Acción ante el Cambio Climático de Chiapas, publicado en 2011 (Secretaría de Medio Ambiente e Historia Natural del Estado de Chiapas [Semahn], 2011), incluye un Inventario Estatal de Gases de Efecto Invernadero (Iegei) con año base 2005. Este Iegei mostró que las emisiones de gases de efecto invernadero (GEI) en el estado se concentran principalmente en el sector Uscusys, constituyendo 59\% de las emisiones totales del estado (16 447 $\mathrm{Gg} \mathrm{CO}_{2}$; de Jong et al., 2010). Estas emisiones proceden, fundamentalmente, de la conversión de bosques a tierras agrícolas y pastizales (potreros) y de la degradación forestal. El segundo sector con mayores emisiones fue el agrícola (5392 Gg $\mathrm{CO}_{2}$; Jiménez et al., 2010; 19\% de las emisiones totales), principalmente por la fermentación entérica del ganado bovino y la fertilización nitrogenada de los suelos agrícolas. Estos datos explican la importancia para el estado de diseñar e implementar un mecanismo REDD+ con un enfoque de desarrollo rural sostenible bajo en emisiones, que responda a las necesidades propias de mitigación y a la realidad estatal, aunque aunado a los esfuerzos nacionales (Semahn, 2017).

Por otra parte, los datos obtenidos por el Sistema Nacional de Monitoreo Forestal para el estado indican que en Chiapas se han deforestado 991776 ha entre 1993 y 2011 y, en el mismo periodo, se han degradado 599576 ha de bosques primarios (Comisión Nacional Forestal [Conafor], 2016). Estas cifras hacen de Chiapas el estado con mayor deforestación y degradación forestal de los cinco estados incluidos en la Iniciativa de Reducción de Emisiones Nacional (Conafor, 2016). Las tasas de pérdida de la cobertura de bosque detectadas en el estado han sido analizadas, además, en varios estudios. Vaca, Golicher, Cayuela, Hewson y Steininger (2012) documentaron la pérdida de 176438 ha de bosques en el periodo 19902006. Por otra parte, Paz, Marín, Medrano, Ibarra y Pascual (2010) estimaron que la degradación forestal en el estado, definida como el paso de bosques conservados (cobertura $>30 \%$ ) a bosques degradados (cobertura entre $10 \%$ y 30\%), ascendió a 769295 ha entre 1990 y 2009 . Estos mismos autores estimaron que la deforestación entre 1990 y 2009 fue de 577621 ha. Estudios realizados utilizando el mapa de deforestación de Hansen et al. (2013) señalan que en el estado se han perdido 303600 ha entre 2001 y 2012 (Earth Innovation Institute, 2015). A pesar de los diferentes resultados obtenidos por estos trabajos, es claro que la deforestación y degradación forestal son problemas vigentes en el estado.

En cuanto a las causas directas de la deforestación asociadas al uso del suelo que sustituye al bosque tras la tala, según los datos de Instituto Nacional de Estadística, Geografía e Informática [Inegi]), 70\% de la deforestación en Chiapas está relacionada con la actividad ganadera, principalmente con la práctica de la ganadería extensiva (Covaleda, Ranero y Aguilar, 2014). Por otra parte, la deforestación por agricultura se ha relacionado con la agricultura de subsistencia (maíz y frijol de temporal) en todo el estado y con la agricultura comercial (principalmente palma africana) en la región de la Selva Maya (Covaleda et al., 2014).

La mitigación de emisiones en el sector Agricultura, Silvicultura y Otros Usos de la Tierra [Afolu, por sus siglas en inglés], además de los beneficios para el clima en términos de carbono capturado o reducción de emisiones, puede traer aparejados cobeneficios sociales y ambientales, los 
cuales son normalmente valorados en términos cualitativos. Adicionalmente, las actividades de mitigación adoptadas pueden provocar efectos negativos no planeados sobre las personas o el medio ambiente (disminución de utilidades, riesgos para la seguridad alimentaria o riesgos para la biodiversidad), los cuales han de ser evaluados para un adecuado análisis de alternativas de actuación.

En México, los investigadores del Programa Mexicano del Carbono reconocieron la necesidad de valorar los impactos asociados con las actividades de mitigación a escala local, particularmente en el marco del desarrollo de la Estrategia Nacional REDD+, planteando, con este fin, el diseño de una herramienta sencilla para ser usada por tomadores de decisiones, basada en el marco conceptual de los modelos de estados y transiciones (Westoby, Walker y Noy-Meir, 1989; Stringham, Krueger y Shaver, 2001). Como área piloto se tomó el estado de Chiapas, donde se desarrollaron dos versiones de modelos, que han sido documentados en varias publicaciones (Covaleda, Paz y de Jong, 2012a, b; Paz y Covaleda, 2015; Covaleda, Paz y Ranero, 2016).

Los modelos son capaces de evaluar el impacto, en términos de carbono, de la aplicación de actividades de mitigación de emisiones en el sector Afolu, enfocadas a: la reducción de las emisiones por deforestación y degradación forestal, el incremento de los acervos de carbono forestal, el manejo forestal, la conservación, la forestación/ reforestación y el incremento de los acervos de carbono a través de prácticas agrícolas y pecuarias sostenibles. A su vez, permiten incorporar restricciones a los análisis, acotando los resultados, por ejemplo, a actividades que no supongan una pérdida de utilidades o pérdida de empleo para los productores o identificando las necesidades de subsidio, entre otros. Estos modelos son, por tanto, potentes aliados para el diseño de políticas públicas multiobjetivo (Reyes, Covaleda, Perez y Paz, 2012) y permiten la generación de curvas de abatimiento y escenarios de mitigación a distintas escalas (municipal, regional y estatal). Una curva de abatimiento muestra los impactos de reducción de emisiones de una medida (actividad) y los costos asociados, permitiendo establecer un jerarquización del costo-eficiencia de las distintas medidas de mitigación y, por tanto, la planificación temporal de medidas y el dimensionamiento de los esfuerzos asociados (Clerc, Díaz y Campos, 2013). Los escenarios de mitigación, por su parte, permiten estimar el potencial de mitigación de la aplicación de las actividades o medidas seleccionadas sobre una superficie geográfica dada, pudiéndose efectuar también proyecciones temporales.

\section{OBJETIVOS}

Obtener curvas de costos de abatimiento (unitarias) y escenarios de mitigación (de intervención y potenciales), utilizando los modelos de estados y transiciones desarrollados por los autores de este trabajo en el estado de Chiapas (Paz et al., 2012). Se consideraron diferentes tipos de restricciones con el fin de dar una idea de las potencialidades de esta herramienta en la planificación de políticas y acciones de mitigación y del potencial de mitigación del estado de Chiapas en el sector Afolu, particularmente con actividades REDD+.

\section{MATERIALES Y MÉTODOS}

\section{Área de estudio}

El estado de Chiapas se encuentra situado en el sureste de la República Mexicana, ocupando una superficie de 73 $611 \mathrm{~km}^{2}$, lo que constituye $3.7 \%$ de la superficie nacional (Fig. 1). Chiapas, con su topografía accidentada, variedad de climas e intervalo altitudinal, es poseedor de una de las más grandes riquezas biológicas de México. Su riqueza florística incluye cerca de 10000 especies de plantas vasculares, la cual es solo superada en México por la de Oaxaca (Comisión Nacional para el Conocimiento y Uso de la Biodiversidad [Conabio], 2013). A su vez, el estado cuenta con grandes extensiones de selvas altas perennifolias (633 $503 \mathrm{ha}$ ), bosques mesófilos (231 $777 \mathrm{ha}$ ) y manglares (43 $602 \mathrm{ha}$ ), los cuales acumulan altas cantidades de carbono tanto en su biomasa como en el suelo (Semahn, 2017).

Los procesos de cambio de uso del suelo en el territorio están influenciados por distintos factores, entre los que destacan el clima, la vegetación, el relieve y el poten- 


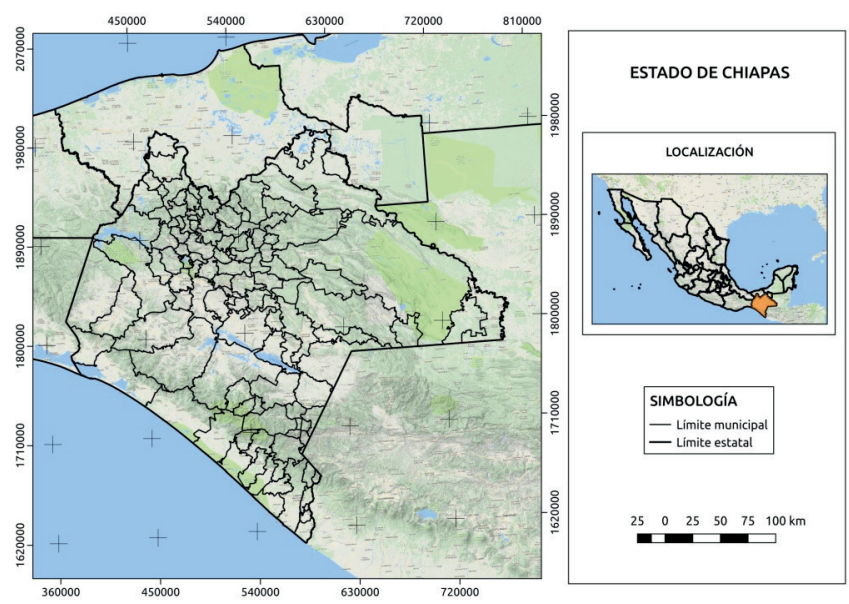

Figura 1. Localización del estado de Chiapas en la República Mexicana.

cial agropecuario de los suelos, así como la cultura de la población y la economía regional. Por ello, con el fin de entender mejor las dinámicas de cambio de uso del suelo en el estado, Paz et al. (2012) propusieron una regionalización ecológico-fisiográfica, basada en las ecorregiones tipo 1 (Inegi-Conabio-INE, 2009) y la clasificación en regiones fisiográficas de Mullerried (1957); adicionalmente, se tuvieron en cuenta consideraciones administra- tivo-económicas (regiones económicas) y culturales, según se describe en Covaleda (2010). Posteriormente, esta clasificación fue modificada para adaptarse a los límites municipales (Fig. 2).

\section{Modelos de dinámica de cambio de uso del suelo en Chiapas}

Los modelos de estados y transiciones (METs) se componen de tres elementos básicos (Stringham et al., 2001): los estados (tipos de vegetación/usos del suelo), las transiciones (trayectorias de cambio) y los umbrales (puntos en el espacio y tiempo que indican que se ha sobrepasado la capacidad de "auto-reparación" o que, a través de factores humanos, se mantiene en el estado deseado). En Chiapas se han elaborado ocho modelos regionales ( $\mathrm{Paz}$ et al., 2012; Covaleda et al., 2016) basados en la regionalización de la figura 2. Estos modelos se basaron en los principales tipos de vegetación y usos del suelo locales identificados en las distintas regiones y, además, se incluyeron una serie de sistemas productivos sostenibles como los agroforestales (milpa con árboles dispersos, taungya y acahual mejorado) y silvopastoriles (pastizal con árboles dispersos y cercos vivos) que son aplicados en algunas comunidades rurales (Ambio, 2012). Otros, como la labranza de conservación y
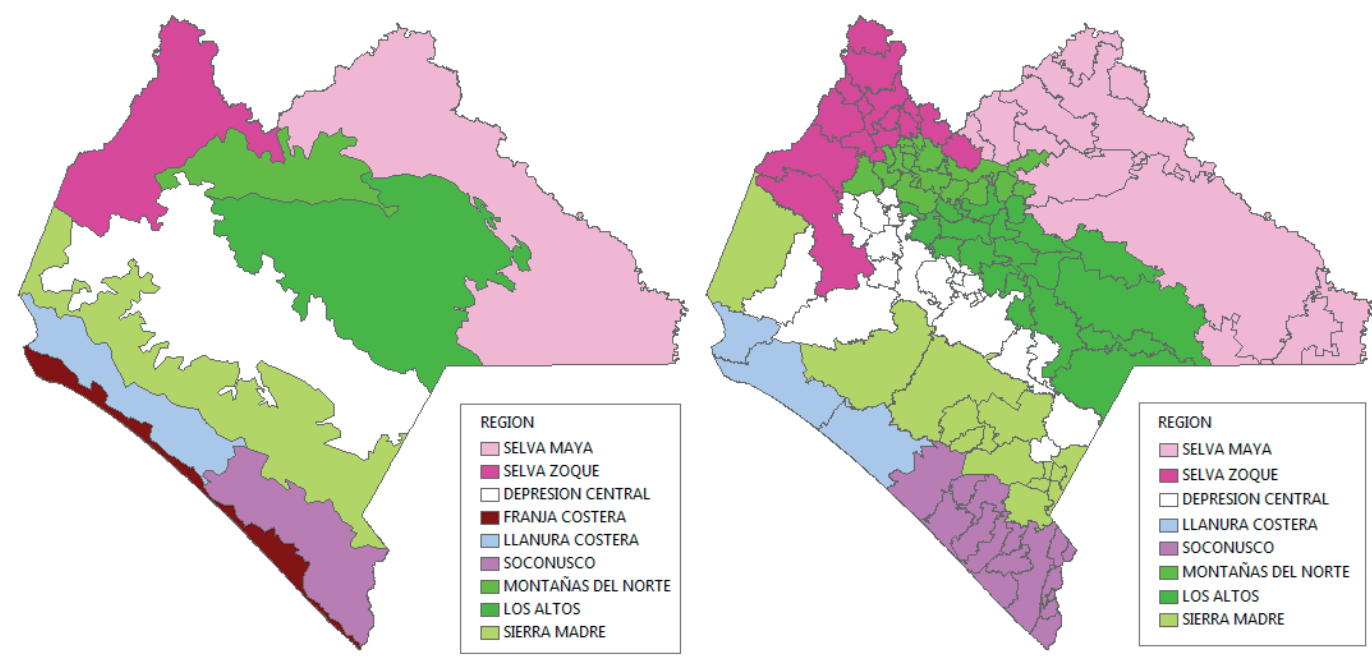

Figura 2. Regionalización propuesta de Chiapas: ecológica-fisiográfica y su modificación municipal. 
el MIAF (maíz intercalado con árboles frutales) constituyen alternativas para mejorar la producción agrícola y, además, contribuyen a la captura de carbono en agroecosistemas (Eagle et al., 2011).

Los estados definidos en cada modelo fueron caracterizados en función de los contenidos promedio de carbono (C) de los almacenes de biomasa viva y suelo (hasta $30 \mathrm{~cm}$ de profundidad) (Covaleda, Paz y de Jong, 2013) y, además, de variables económico-financieras (valor presente neto, número de jornales, monto de crédito requerido y monto de los subsidios disponibles). En la estimación del valor presente neto (VPN) se consideraron tres tasas de interés (TI): 6\%, 12\% y 18\%, para conocer el efecto del valor del dinero en el tiempo. Además, se calcularon los costos de oportunidad de las actividades, ya que con este dato es posible analizar la diferencia en la rentabilidad neta entre usos alternos del suelo (transición entre estados). En el caso de REDD+, estos costos se refieren a la diferencia entre conservar o mejorar los bosques versus convertirlos a otros usos del suelo, generalmente más rentables (White y Minang, 2011). Estos se calculan dividiendo la diferencia de rentabilidad entre un estado final y otro inicial (expresada como valor presente neto) por la diferencia en las reservas de carbono entre el estado final e inicial.

Estos modelos permiten, por tanto, conocer los impactos en términos de las variables consideradas de pasar de un estado inicial A hacia otro B. En los modelos desarrollados en Chiapas, la primera versión incluyó únicamente el carbono (Covaleda, 2010); después se incorporaron variables económico-financieras (Paz et al., 2012) y, en una posterior actualización de los modelos de algunas regiones, se llegaron a incluir indicadores socio-económicos, restricciones asociadas a distintos tipos de productores y actividades elegibles en los mercados de carbono (Covaleda, 2014). La transición entre estados, por tanto, puede o no suponer el mejoramiento de los almacenes de carbono del sistema y, además, hay que evaluar los costos e impactos socio-económicos asociados con el paso de un estado a otro (Fig. 3).

Por ejemplo, los METs pueden estimar los impactos sociados a la deforestación de un bosque mesófilo (Estado A) por la implantación de un pastizal (Estado B), lo cual generará pérdidas de carbono o el establecimiento de una plantación forestal (Estado B) sobre una milpa abandonada (Estado A).

Estos modelos permiten, además, simular la dinámica del $\mathrm{C}$ y variables económico-financieras en términos anuales. Para el carbono se utilizó un modelo de crecimiento sigmoide asimétrico (Yin, Gourdriaan, Lantinga, Vos y Spiertz, 2003; Paz, Covaleda y de Jong, 2015) por su simplicidad, debido a que depende únicamente de un parámetro y a que se ajusta bien a los valores experimentales, ya que aproxima relaciones más complejas usadas en la práctica forestal. El valor presente neto y las variables económico-financieras asociadas a cada estado fueron proyectadas a 30 años, de acuerdo con los lineamientos del Banco Mundial (White y Minang, 2011) y la duración de los contratos con la tenencia de la tierra del sector social definidos en la Ley Agraria mexicana. De esta manera es posible generar curvas de costos de abatimiento y plantear escenarios de mitigación para el sector rural de distintos

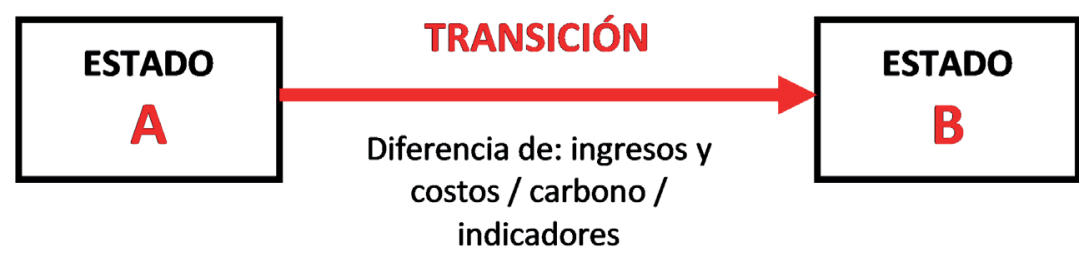

FIgURA 3. Transición entre estados. 
tipos (potenciales, de intervención). Los modelos utilizados en este trabajo son los desarrollados por Paz et al. (2012) y los resultados mostrados son los promedios obtenidos tras proyectar las variables (e.g., biomasa o carbono) a 30 años (usando los modelos definidos). Los análisis que permiten plantear los modelos pueden centrarse en distintos grupos de actividades genéricas, pudiéndose seleccionar distintos componentes (Tabla 1).

Adicionalmente, los análisis pueden acotarse incluyendo restricciones relacionadas con el efecto sobre el empleo y la disponibilidad de subsidio y crédito, así como con los valores de los costos de oportunidad (Tabla 2).

Los efectos sobre el empleo se evalúan calculando la diferencia de jornales entre un estado final y otro inicial, los cuales normalmente no requieren el mismo esfuerzo de trabajo.

Los créditos, en el estudio, se dividen en refaccionarios, que se conceden por periodos superiores a 2 años y de habilitación/avío, para periodos menores a dos años. En este sentido, se diferencia la situación de poner en marcha una actividad con el apoyo de un crédito o sin él. Aunque

TAвLA 1. Grupos de actividades y sus componentes que pueden seleccionarse para análisis con los modelos.

\begin{tabular}{cc}
\hline Grupo de actividades & Componentes \\
\hline REDD+ & Deforestación (DF) \\
& Degradación forestal (DG) \\
& Incremento de almacenes de carbono (IA) \\
& Conservación (CS) \\
Morestación/Reforestación (AR) & Incremento de almacenes de carbono (IA) \\
\hline Agropecuario & Degradación (DG) \\
& Incremento de almacenes de carbono (IA) \\
& Todas las componentes (GRAL) \\
\hline
\end{tabular}

TавLA 2. Restricciones para el análisis.

\begin{tabular}{cc}
\hline Restricción & Variables asociadas \\
\hline Efecto sobre el empleo & $\begin{array}{r}\text { Sin pérdida de empleo, diferencia de jornales positiva (JP) } \\
\text { Con pérdida de empleo, diferencia de jornales negativa (JN) }\end{array}$ \\
\hline Efecto del crédito & Si hay crédito (SC) \\
Efecto del subsidio & No hay crédito (NC) \\
\hline Signo del costo de oportunidad & Actividades con subsidio (CS) \\
\hline Valor del costo de oportunidad & Actividades sin subsidio (SS) \\
\hline
\end{tabular}


conviene señalar que no todas las actividades incluidas en los modelos tienen acceso a crédito, por ser marginales o por no cumplir con estándares de financiamiento bancario.

En el caso de proyectos con subsidio se consideraron los apoyos federales (e.g., Programa de Apoyos Directos al Campo [Procampo] y Programa de Producción Pecuaria Sustentable y Ordenamiento Ganadero y Apícola [Progan] de la Secretaría de Agricultura, Ganadería, Desarrollo Rural, Pesca y Alimentación [Sagarpa]) y estatales (e.g., apoyos de la Secretaría del Campo del Gobierno de Chiapas). Los programas de pagos por servicios ambientales (e.g., PSA de la Conafor) se consideraron como subsidios, dado que no están basados en resultados y no son verificables.

En el caso de los costos de oportunidad, es importante conocer su signo (positivo/negativo) y la situación relativa con respecto a las demás actividades evaluadas; así, los costos de oportunidad mínimos se refieren a transiciones donde el costo asociado es el menor de las opciones analizadas. En el caso del promedio, el proyecto o transición es aquel que se acerca más al valor promedio de los costos de oportunidad de los múltiples estados finales.

\section{Construcción de curvas de costos de abatimiento}

La construcción de curvas de costos de abatimiento (White y Minang, 2011; Australian Government, 2011) requiere dos tipos de datos: el potencial de mitigación unitario de las actividades propuestas ( $\mathrm{t} \mathrm{CO}_{2} \mathrm{ha}^{-1}$ ) y los costos de oportunidad (USD/t $\mathrm{CO}_{2}^{-1}$ ).

Para desarrollar las curvas de abatimiento utilizando la información asociada con los METs regionales de Chia- pas, se generaron matrices de transición entre estados (del estado inicial al final) por regiones para las variables presentadas en la tabla $3 \mathrm{y}$, posteriormente, con base en esta información, se calcularon los costos de oportunidad y se elaboraron las curvas.

Este trabajo se centró en el análisis de costos de oportunidad positivos y negativos (White y Minang, 2011). Los costos de oportunidad negativos están asociados con la situación donde, reduciendo una actividad (emisiones), se generan ganancias netas y no costos. Las estimaciones de costos de oportunidad (USD/t $\mathrm{CO}_{2}^{-1} \mathrm{ha}^{-1}$ ) fueron realizadas para los casos con subsidio y sin subsidio y, en cada uno de ellos, se evaluaron las situaciones de TI de 6\%, $12 \%$ y $18 \%$. El caso con subsidio y TI $=12 \%$ se considera como el normal o de referencia para los análisis de sensibilidad.

Las curvas de costos de abatimiento generadas en este trabajo se centraron en el grupo de actividades REDD+ (todos sus componentes) y en transiciones asociadas con procesos de deforestación y degradación forestal, considerando la situación con subsidio. Entre las restricciones posibles de evaluación, se seleccionó el efecto sobre el empleo.

Los análisis que se muestran a continuación representan condiciones para el año 2012, fecha de realización del estudio, por lo que la paridad peso-dólar fue de MXN 12/ USD. Considerando el tipo actual de cambio, los valores mostrados pueden considerarse como conservadores, donde los costos de oportunidad establecidos serían menores. De cualquier forma, la paridad peso-dólar debe ser actualizada cada vez que se utilicen los modelos, para

TABla 3. Variables utilizadas en la construcción de curvas de costos de abatimiento.

\begin{tabular}{cc}
\hline Potencial de mitigación unitario $\left(\mathrm{CO}_{2} \mathrm{ha}^{-1}\right)$ & Diferencia de rentabilidad $\left(\right.$ USD ha $\left.{ }^{-1}\right)$ \\
\hline -Diferencia del carbono en la biomasa viva aérea & \\
-Diferencia del carbono en la biomasa viva subterránea & -Diferencia del valor presente neto \\
-Diferencia del carbono orgánico del suelo & \\
-Diferencia del carbono total & \\
-Diferencia total de toneladas de $\mathrm{CO}_{2}$ equivalente por hectárea &
\end{tabular}


obtener los resultados más ajustados a la realidad. Por ejemplo, si la paridad a la fecha es MXN 20/USD, los resultados mostrados en este trabajo deben multiplicarse por 20/12 para tener datos actualizados.

A pesar de que los modelos fueron desarrollados a escala regional (un modelo por región), es posible particularizar los resultados a escala municipal, ya que la regionalización utilizada para estos análisis respeta los límites administrativos municipales, y los modelos, en esta versión, no son espacialmente explícitos; es decir, se pueden usar los modelos regionales empleando información a escala municipal. Por otra parte, para obtener información a escala estatal, es posible generar matrices de transición de los promedios de todas las matrices regionales.

\section{Impactos por cambio de uso del suelo y} generación de escenarios de intervención regionales

La selección de los estados iniciales y finales de los modelos permite realizar diferentes tipos de análisis, entre los que destacan el análisis de impactos por cambio de uso del suelo y el planteamiento de escenarios de mitigación para planear acciones de intervención. Los impactos pueden deberse a la degradación forestal y a la deforestación con fines agrícolas y pecuarios.

Los escenarios, por su parte, pueden centrarse en distintos sectores: forestal, agrícola y pecuario, permitiendo visualizar los impactos de implementar diferentes sistemas sostenibles o prácticas de manejo en un determinado municipio o región.

En el presente trabajo se tomó como ejemplo el caso de la deforestación de una selva alta perennifolia en la región Selva Maya para restablecer diferentes usos del suelo locales. A partir de ahí, se plantearon escenarios para mejorar la captura de carbono en el sector agropecuario de esa región.

\section{Generación de escenarios de mitigación potenciales}

Los escenarios generados representan el potencial de mitigación total para una unidad territorial determinada (estado, región, municipio) de una serie de actividades (REDD+, AR, agropecuarias) en función de sus costos de oportunidad (USD/t $\mathrm{CO}_{2}^{-1}$ ).

Este análisis requiere el conocimiento de la superficie de los distintos tipos de vegetación y usos del suelo de las unidades geográficas consideradas. En este trabajo los tipos de vegetación y usos del suelo utilizados en los METs fueron homologados con las clases de la serie IV (año base 2007) del mapa de uso del suelo y vegetación del Inegi. Sin embargo, la homologación de los usos del suelo locales (utilizados en los METs) con los sistemas de clasificación genéricos de uso del suelo y vegetación utilizados en México, particularmente los del Inegi, presenta problemas de asignación de "uno a muchos", los cuales han sido discutidos en Paz et al. (2012), Paz (2015) y Covaleda et al. (2016).

Adicionalmente, se utilizó el mapa de probabilidad de deforestación generado por Castillo et al. (2010) para el Programa de Acción Ante el Cambio Climático del estado de Chiapas [Paccch] (Semahn, 2011) con el fin de determinar las superficies promedio esperadas deforestadas/degradadas, para así poder determinar los costos de oportunidad asociados y construir escenarios de mitigación. La figura 4 muestra las probabilidades de deforestación en Chiapas según este mapa. La probabilidad más alta detectada fue de 0.54 .

La información de las superficies en riesgo de deforestación y degradación a escala regional fue introducida en los modelos con el fin de generar escenarios de mitigación. En los análisis siguientes solo se consideraron áreas dentro de Chiapas con probabilidades mayores a cero de riesgo de deforestación.

$\mathrm{Al}$ igual que con las curvas de abatimiento, es posible generar escenarios a diferentes escalas (estatal, regional y municipal). En total, a escala estatal se evaluaron 1114 transiciones o proyectos que se transformaron en 1536 al asignar estados múltiples a los usos del suelo considerados por el Inegi (problema de asignación de "uno a muchos"). En la escala regional, las transiciones evaluadas fueron 3625 que se propagaron a 4186 por el problema de asignación de "uno a muchos". Para el caso 


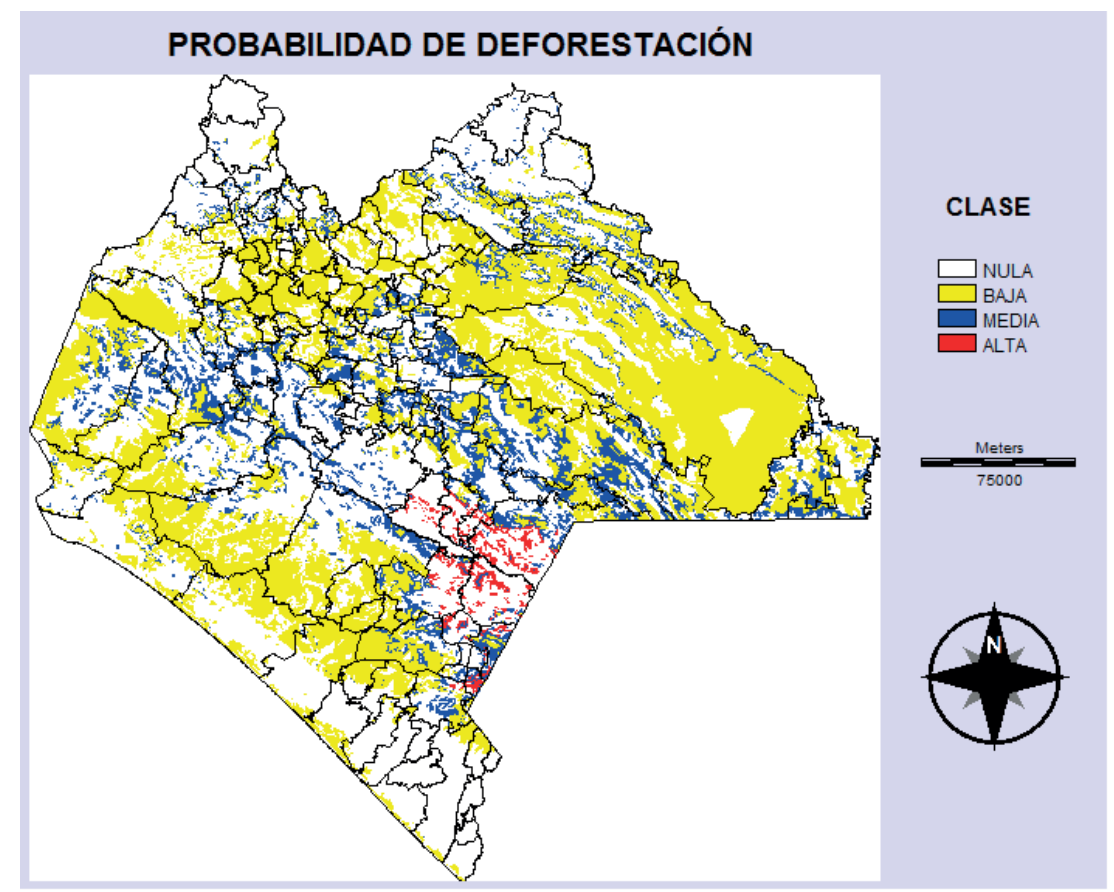

Figura 4. Probabilidades de deforestación en Chiapas, donde las clases están definidas por los siguientes intervalos: Nula = 0.0, Baja $=0.0-0.2$, Media = $0.2-0.4$, Alta = $0.4-0.6$ (Fuente: adaptado de Castillo et al., 2010).

de los municipios, las transiciones o proyectos evaluados superaron los 80000 .

En este trabajo, sin embargo, por motivos de espacio, se presentan resultados obtenidos para REDD+y sector agropecuario y a escala estatal. Las restricciones (condiciones a cumplir) asociadas con las actividades propuestas para conseguir la mitigación de emisiones en el sector Afolu en Chiapas fueron todas las incluidas en la tabla 2.

Para el cálculo de los estadísticos de los costos de oportunidad, se definió un valor umbral máximo de USD $20 / \mathrm{CO}_{2}^{-1}$ para los valores positivos y un valor umbral mínimo de -USD 100/t CO ${ }_{2}^{-1}$ para los negativos (menores $o$ iguales a cero). Entre las actividades (o transiciones) evaluadas se identificaron algunas altamente redituables (e.g., proyectos forestales) y otras muy marginales (e.g., milpas de maíz a nivel de subsistencia). El acotar el intervalo de actividades a los valores definidos permitió concentrarse solo en los proyectos con costos de oportunidad razonables para implementar políticas públicas.

\section{RESUlTADOS}

\section{Curvas de costos de abatimiento para REDD+ en Chiapas}

En este apartado se presentan los resultados obtenidos a escala estatal para REDD+, considerando todos sus componentes por una parte y, por otra, la deforestación y la degradación forestal.

La figura 5 muestra la curva de costos de abatimiento en términos unitarios $\left(\mathrm{t} \mathrm{CO}_{2} \mathrm{ha}^{-1}\right.$ ) para REDD+ (considerando todos sus componentes), para el caso de actividades con subsidio y $\mathrm{TI}=12 \%$, en relación con los potenciales de mitigación para las distintas transiciones evaluadas.

En la figura 6 se muestran las curvas de costos de abatimiento unitario para los componentes de deforestación y degradación forestal, para el caso con subsidio y TI = $12 \%$.

Es importante analizar los efectos en el empleo (jornales) asociados con los cambios de actividad (transiciones entre estados). La figura 7 muestra el caso de puesta en 

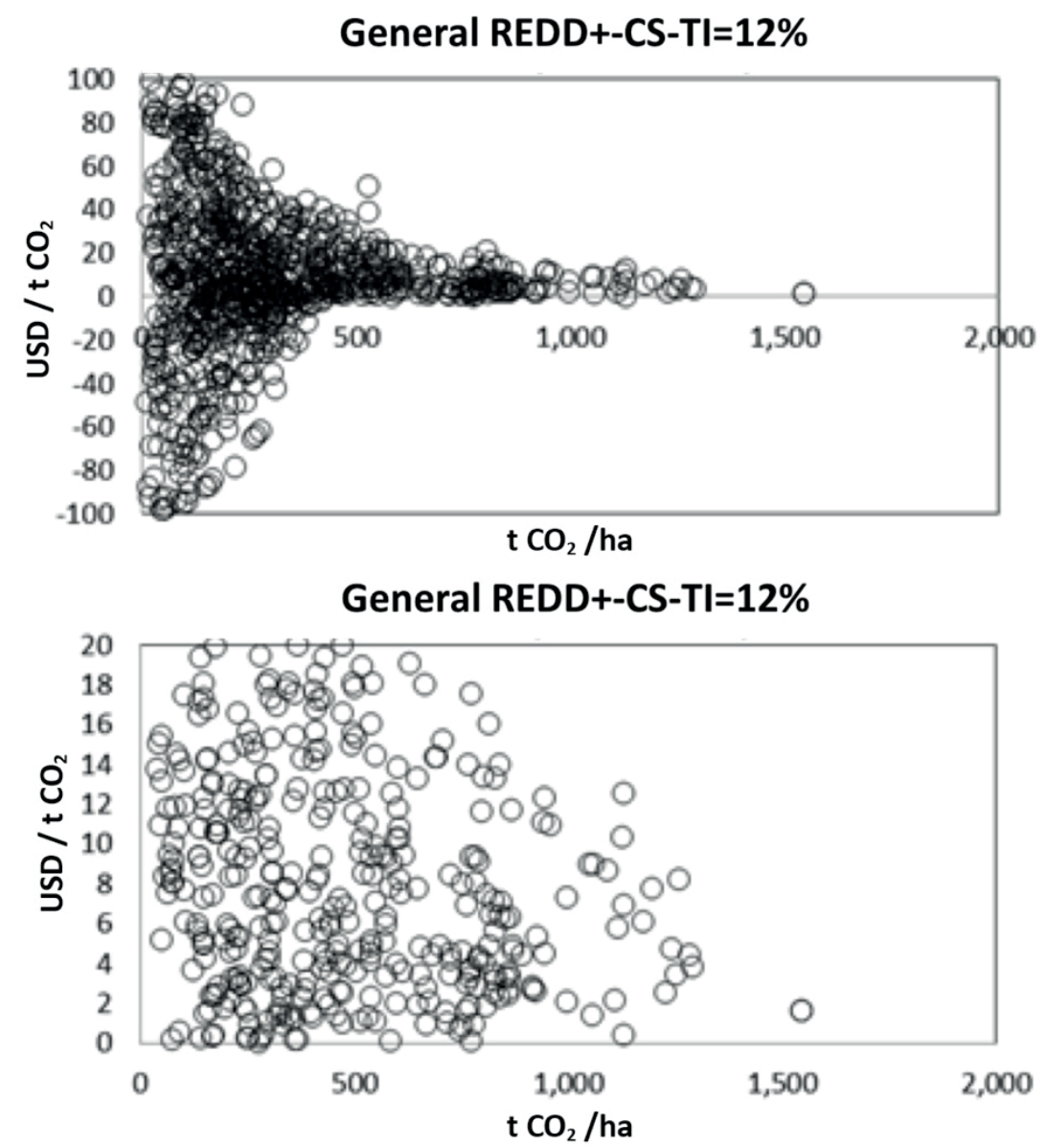

Figura 5. Curva de costos de abatimiento unitarios para REDD+ (todos sus componentes); CS: caso con subsidio y TI (tasa de interés) $=12 \%$, para el estado de Chiapas.

marcha de actividades REDD+ (considerando todos los componentes), con subsidio y $\mathrm{TI}=12 \%$. Esta figura permite observar que muchas transiciones generan pérdidas de empleo, por lo que este criterio debe considerarse en la evaluación de escenarios de mitigación.

\section{Impactos por cambio de uso del suelo y escenarios de intervención regionales}

En la tabla 4 se muestra un ejemplo de los impactos de la transformación de una selva alta perennifolia (sensu Inegi) en la región Selva Maya en términos de carbono total (biomasa viva y suelo), costos de oportunidad (sin subsidio y con subsidio), jornales y acceso a crédito.

La tabla 4 muestra que la degradación forestal y la deforestación de las selvas perennifolias implican la pér- dida de carbono del sistema, particularmente en el caso de la deforestación hacia sistemas productivos agrícolas y pecuarios. En todos los casos analizados, los costos de oportunidad resultaron positivos, lo que implica que los estados finales (usos productivos) resulten más redituables, de manera particular la extracción no regulada de madera. A su vez, todos los usos productivos suponen más empleo o jornales de trabajo para su manejo y aprovechamiento. En relación con el acceso a crédito, las actividades pecuarias y la producción de café pueden beneficiarse de los créditos otorgados por las instituciones financieras regionales para su puesta en marcha.

Otra potencialidad de esta herramienta es la posibilidad de plantear escenarios para capturar carbono e incentivar prácticas sostenibles. En la tabla 5 se muestran 

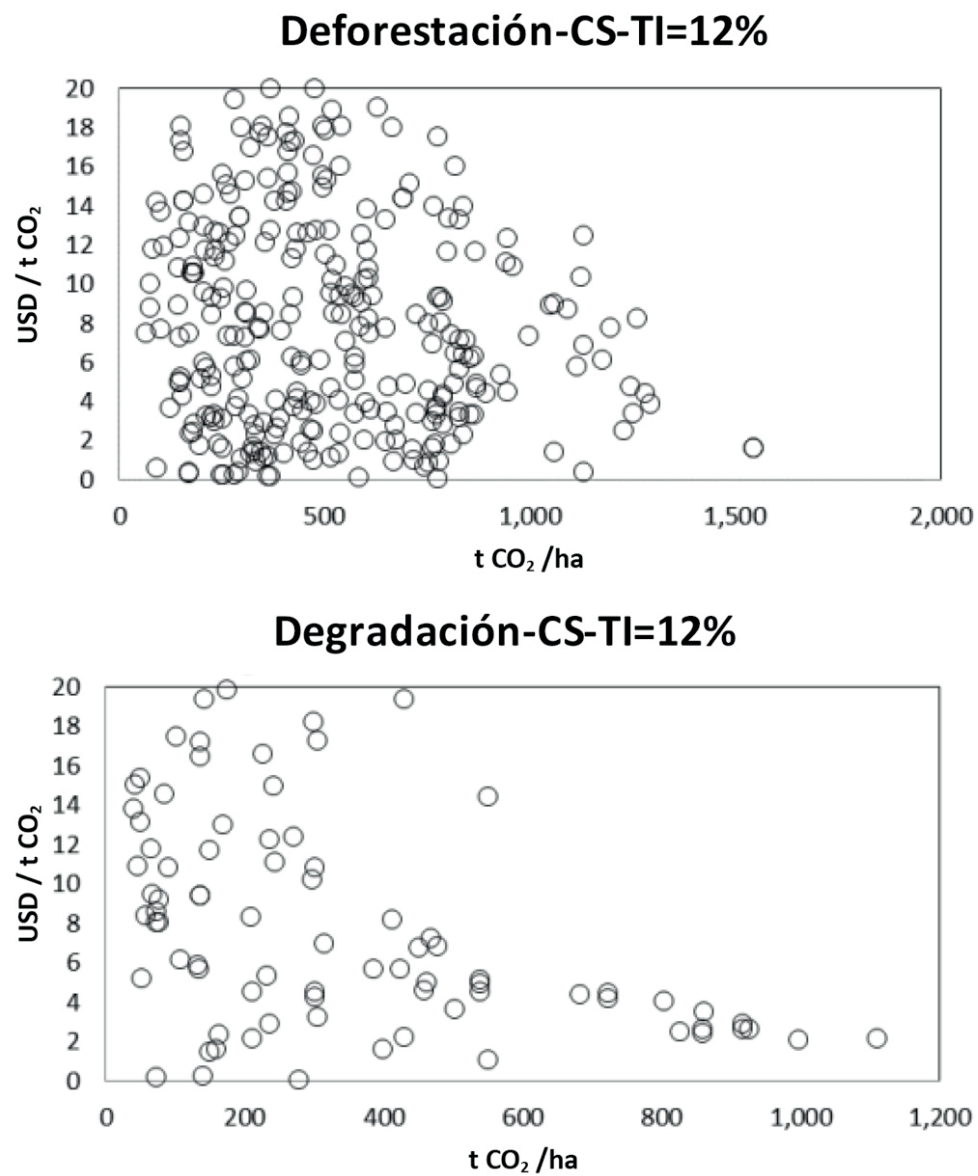

Figura 6. Curva de costos de abatimiento unitarios para los componentes de deforestación y degradación forestal; CS: caso con subsidio y TI (tasa de interés) = 12\%, para el estado de Chiapas.

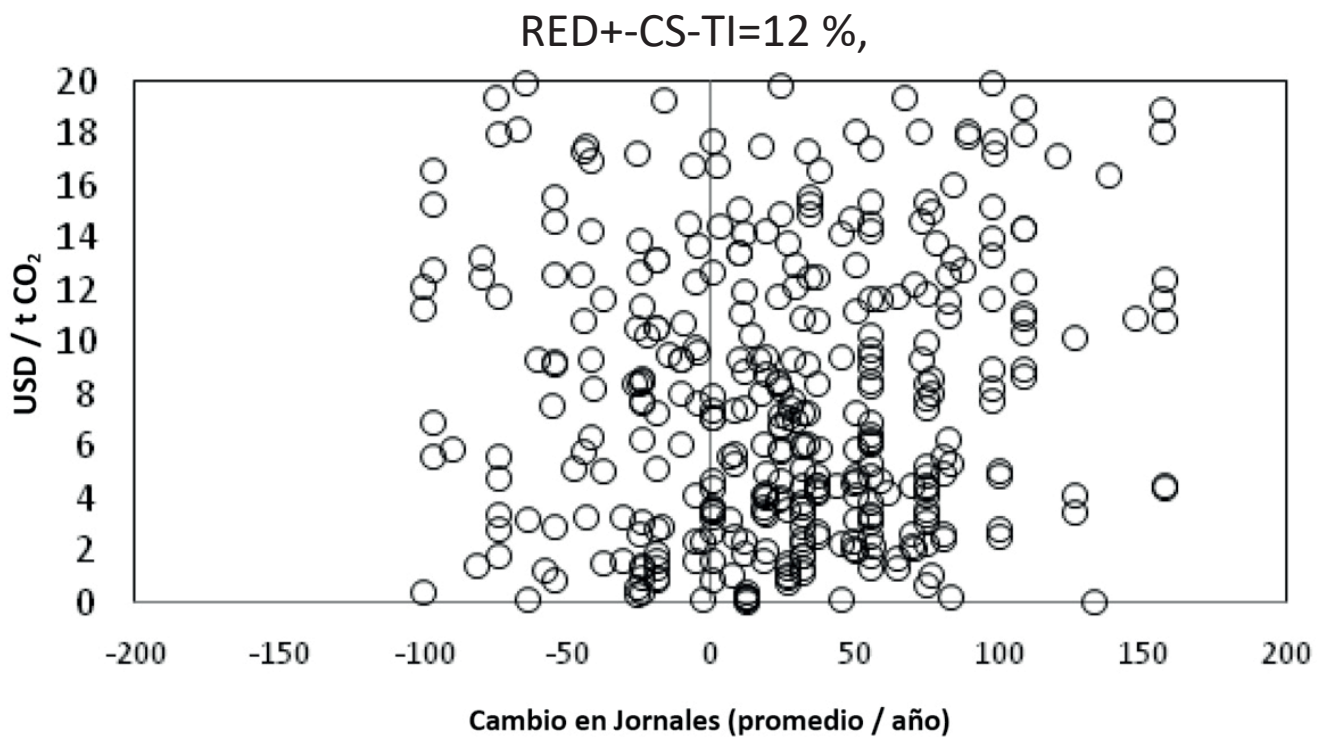

Figura 7. Cambios en jornales producto de la transición entre estados o cambios de actividades en relación con los costos de oportunidad; CS: caso con subsidio y TI (tasa de interés) $=12 \%$, para el estado de Chiapas. 
TAвla 4. Impactos sobre el carbono por la transición de una selva alta perennifolia en la región Selva Maya de Chiapas a usos agrícolas, pecuarios y estados de bosque más degradado; costos de oportunidad, efecto sobre el empleo y acceso a crédito.

\begin{tabular}{|c|c|c|c|c|c|c|}
\hline Estado inicial & Estado final & $\begin{array}{c}C_{\text {TOT }} \\
\left(เ \mathrm{CO}_{2} h a^{-1}\right)\end{array}$ & $\begin{array}{l}\text { Costo oportunidad-SS } \\
\text { (USD ha-1) }\end{array}$ & $\begin{array}{l}\text { Costo oportunidad-CS } \\
\text { (USD ha-l) }\end{array}$ & $\begin{array}{l}\text { Diferencia de Jornales } \\
\text { (días año }{ }^{-1} \text { ) }\end{array}$ & $\begin{array}{c}\text { Acceso a } \\
\text { crédito }\end{array}$ \\
\hline \multirow{3}{*}{ SP } & P-troph & -1314.7 & 0005.5 & 0007.1 & 0097.0 & Si \\
\hline & AP-MF & -1370.5 & 0003.1 & 0003.3 & 0055.0 & No \\
\hline & Al-MF-troph & -0975.5 & 0004.0 & 0004.3 & 0074.0 & No \\
\hline SP & SPd-m & -0906.3 & 0019.1 & 0018.7 & 0080.0 & No \\
\hline SP & $\mathrm{CN}$ & -1028.1 & 0002.9 & 0002.6 & 0100.0 & Si \\
\hline
\end{tabular}

$\mathrm{C}_{\text {ro: }}$ carbono de la biomasa viva y suelo (profundidad: $\mathrm{O} \mathrm{cm}$ - $30 \mathrm{~cm}$ ); SS: sin subsidio; CS: con subsidio; SP: selva perennifolia; P-troph: pastizal tradicional en zona tropical húmeda; PA-troph: pastizal con árboles en zona tropical húmeda; AP-MF: agricultura permanente maíz-frijol; Al-MF-troph: agricultura itinerante maíz-frijol en zona tropical húmeda; SPd-m: selva perennifolia degradada por madera; CN: café natural; CO: café orgánico. (Fuente: Paz et al., 2012).

TABLA 5. Impacto sobre el carbono de la aplicación de prácticas sostenibles agrícolas y pecuarias; costos de oportunidad, efecto sobre el empleo y acceso a crédito.

\begin{tabular}{|c|c|c|c|c|c|c|}
\hline $\begin{array}{c}\text { Estado } \\
\text { inicial } \\
\end{array}$ & $\begin{array}{c}\text { Estado } \\
\text { final }\end{array}$ & $\begin{array}{c}C_{\text {TOT }} \\
\left(เ \mathrm{CO}_{2} h a^{-1}\right) \\
\end{array}$ & $\begin{array}{c}\text { Costo oportunidad-SS } \\
\text { (USD ha-1) }\end{array}$ & $\begin{array}{c}\text { Costo oportunidad-CS } \\
\text { (USD ha-1) }\end{array}$ & $\begin{array}{l}\text { Diferencia de Jor- } \\
\text { nales (días año-1) }\end{array}$ & $\begin{array}{c}\text { Acceso a } \\
\text { crédito }\end{array}$ \\
\hline \multirow{2}{*}{ P-troph } & PA-troph & 094.50 & -000.27 & -000.27 & 011.00 & $\mathrm{Si}$ \\
\hline & PFo-trop & 353.40 & -011.70 & -018.50 & -087.00 & $\mathrm{Si}$ \\
\hline \multirow{2}{*}{ AP-MF } & MIAF-limón & 062.60 & -052.00 & -061.00 & 055.00 & $\mathrm{Si}$ \\
\hline & LC & 029.90 & -013.00 & -007.00 & -026.00 & No \\
\hline $\mathrm{CN}$ & $\mathrm{CO}$ & 215.70 & -002.76 & -002.76 & 057.00 & $\mathrm{Si}$ \\
\hline
\end{tabular}

$\mathrm{C}_{\text {to: }}$ carbono de la biomasa viva y suelo (profundidad: $\mathrm{O}$ cm - $30 \mathrm{~cm}$ ); SS: sin subsidio; CS: con subsidio; P-troph: pastizal tradicional en zona tropical húmeda; PA-troph: pastizal con árboles en zona tropical húmeda; PFo-trop: plantación forestal tropical; AP-MF: agricultura permanente maíz-frijol; MIAF-limón: maíz intercalado con limón; LC: Iabranza de conservación; CN: café natural; CO: café orgánico. (Fuente: Paz et al., 2012).

algunas opciones para incrementar la captura de carbono, a partir de prácticas agrícolas y pecuarias convencionales.

Como se observa en la tabla 5 , todas las prácticas sostenibles planteadas suponen un incremento de los almacenes de carbono con respecto a los estados iniciales y los costos de oportunidad asociados son negativos. En este caso, la liberación de áreas de potrero para el establecimiento de plantaciones forestales es la práctica que más carbono permite capturar, seguida de la transformación de cafetales naturales o rústicos (escaso manejo y escasa utilización de insumos externos) en cafetales orgánicos (según la descripción de estados incluida en Paz et al., 2012). En términos de empleo, los potreros con árboles, el MIAF (maíz intercalado con árboles frutales) y el cafetal orgánico necesitan más mano de obra que los sistemas convencionales iniciales. Además, la mayoría de las prácticas sostenibles analizadas podrían ser apicadas bajo algún esquema de crédito disponible.

\section{Escenarios de mitigación potenciales para REDD+} y sector agropecuario en Chiapas (superficie en riesgo de deforestación)

En este apartado se presentan algunos de los escenarios de mitigación obtenidos para el estado de Chiapas, donde se consideraron diferentes factores condicionantes para su desarrollo.

\section{Escenarios de mitigación con costos de oportunidad positivos, sin restricciones (VP)}

La figura 8 muestra los escenarios de mitigación generados para costos de oportunidad positivos y sin restricciones asociadas, para el caso con subsidio y $\mathrm{TI}=12 \%$. 


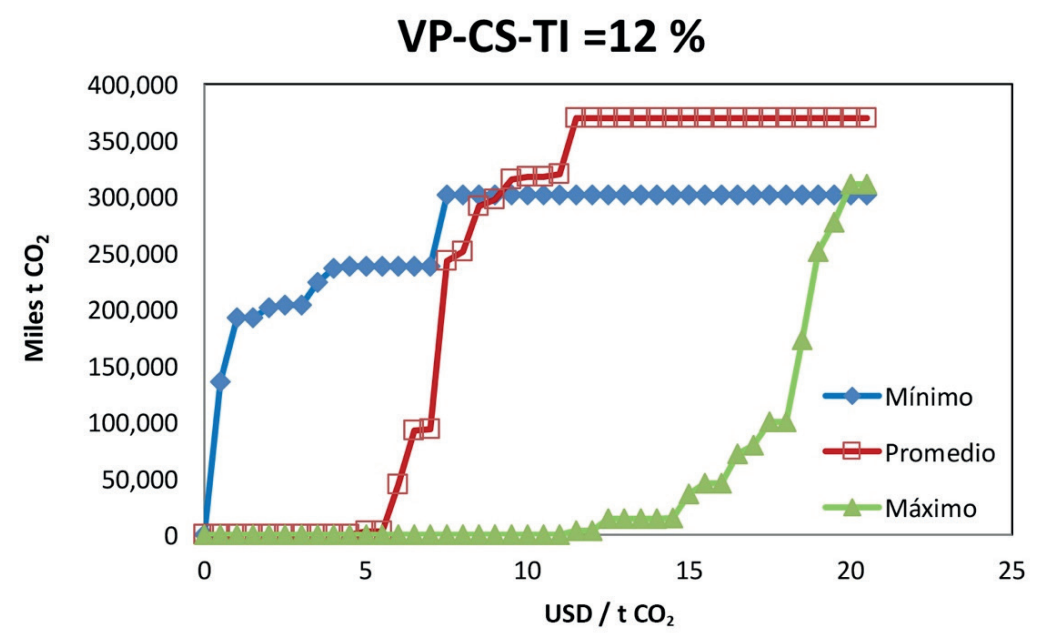

Figura 8. Efecto del valor del costo de oportunidad para el escenario de mitigación: VP: costos de oportunidad positivos, CS: con subsidio y TI (tasa de interés) = 12\% (superficies en riesgo de deforestación/degradación).

Cada punto de la figura 8 es una actividad de mitigación (transición) planteada para los sectores agrícola, pecuario o forestal. Los estadísticos mínimo, promedio y máximo se refieren a los valores de carbono para un costo de oportunidad asociado con un estado inicial dado y múltiples estados finales.

En la figura 8 se observa que con costo de oportunidad de USD 1/t $\mathrm{CO}_{2}^{-1}$, el potencial de reducción de emisiones es de 200000000 t $\mathrm{CO}_{2}$ para los casos de solo considerar actividades de costo mínimo. Para costos promedio, este potencial de reducción tiene un costo de USD 7.5/t $\mathrm{CO}_{2}^{-1}$ y USD 19/t $\mathrm{CO}_{2}^{-1}$ para actividades con costos máximos.

La figura 9 muestra la situación de costos de oportunidad positivos promedio, con subsidio, para el efecto de la TI.

En la figura 9 se observa que para un potencial de reducción de emisiones de 200000000 t $\mathrm{CO}_{2}$, el costo es

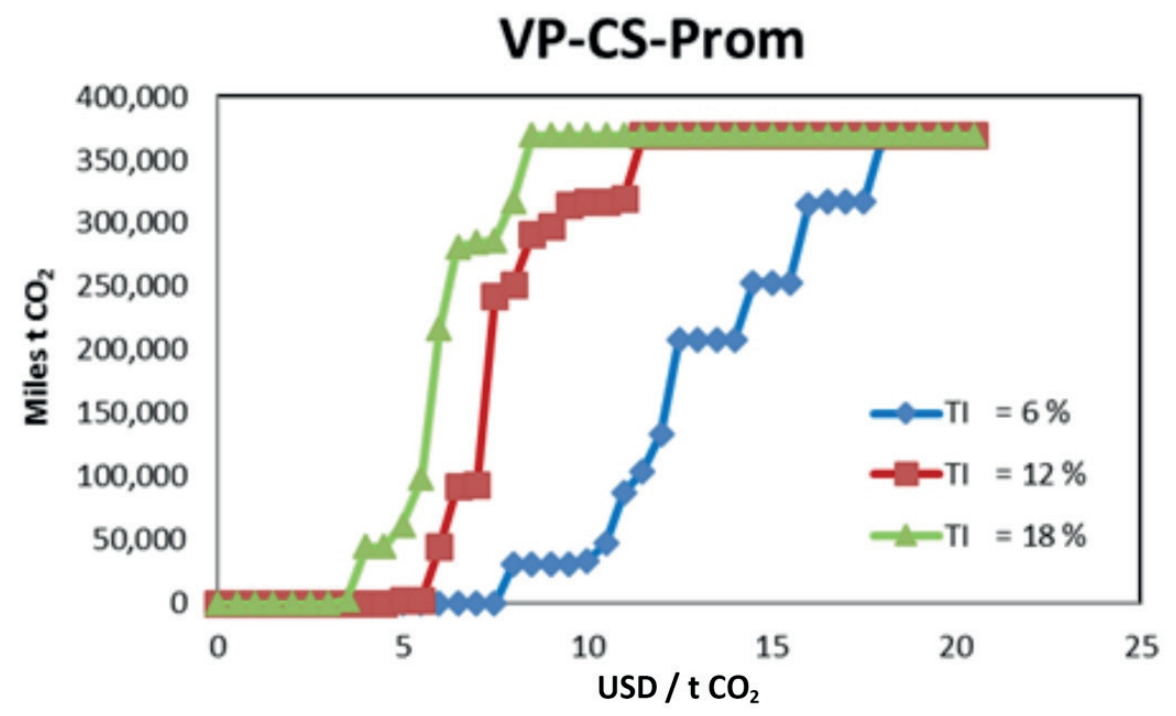

Figura 9. Efecto de la tasa de interés (TI) para el escenario de mitigación: VP: costos de oportunidad positivos, CS: con subsidio; Prom: costos de oportunidad promedio (superficies en riesgo de deforestación/degradación). 
de USD 6/t $\mathrm{CO}_{2}^{-1}$ para una TI de $6 \%$, de USD 7.5/t $\mathrm{CO}_{2}{ }^{-1}$ para una TI de $12 \%$ y USD $12.5 / \mathrm{t} \mathrm{CO}_{2}^{-1}$ para una de $18 \%$, mostrando el efecto del valor del dinero en el tiempo.

La figura 10 permite visualizar el efecto del subsidio en los potenciales de mitigación y sus costos de oportunidad asociados, para la situación de costos de oportunidad promedio positivos con $\mathrm{TI}=12 \%$.

La figura 11 muestra la diferencia de reducción de emisiones para el efecto del subsidio (caso sin subsidio-caso con subsidio) en función del costo de oportunidad. Esta figura muestra que para costos de oportunidad entre USD 2.5/t
$\mathrm{CO}_{2}^{-1}$ y USD 5.0/t CO ${ }_{2}^{-1}$, el subsidio implica una pérdida de potencial de reducción de aproximadamente $100000000 \mathrm{t}$ $\mathrm{CO}_{2}$ y para valores mayores a USD 5.0/t $\mathrm{CO}_{2}^{-1}$, este efecto negativo se reduce hasta ser prácticamente nulo, salvo en un caso alrededor de USD 11/t $\mathrm{CO}_{2}^{-1}$ (proyecto forestal).

\section{Escenarios de mitigación con costos de oportunidad} positivos, sin pérdida de empleo y sin crédito (VP-JP-NC) La figura 12 muestra los escenarios de mitigación para costos de oportunidad positivos, sin pérdida de empleo, sin crédito y $\mathrm{TI}=12 \%$.

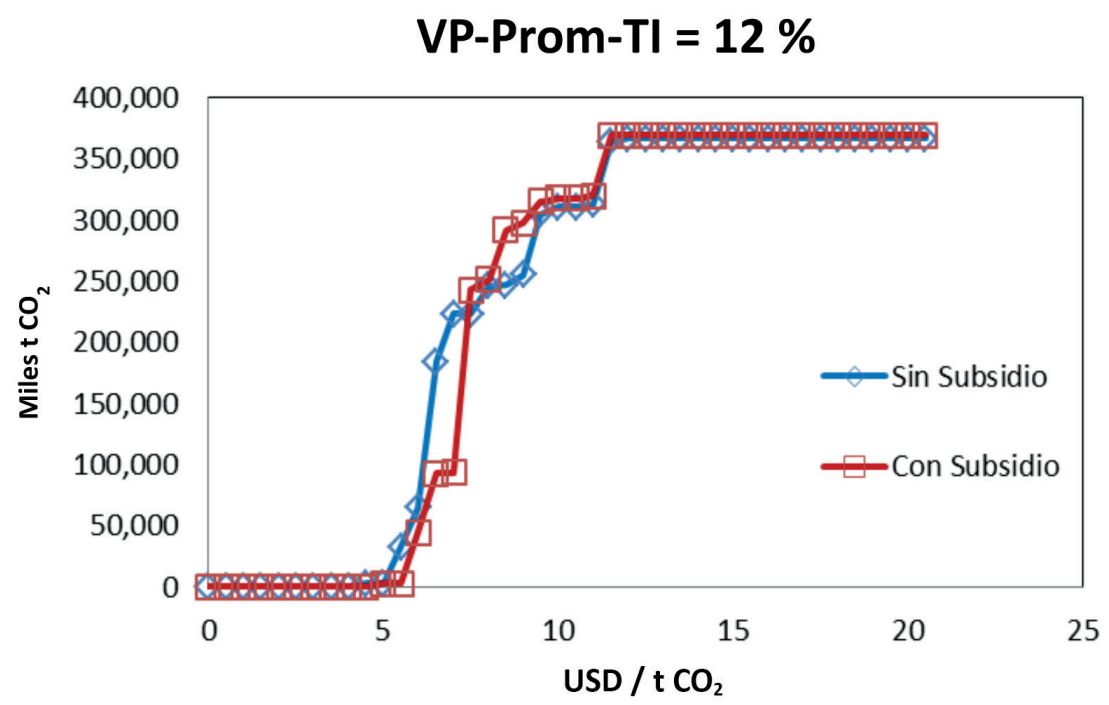

Figura 10. Efecto del subsidio para el escenario de mitigación: VP: costos de oportunidad positivos, Prom: costos de oportunidad promedio y TI (tasa de interés) = 12\% (superficies en riesgo de deforestación/degradación).

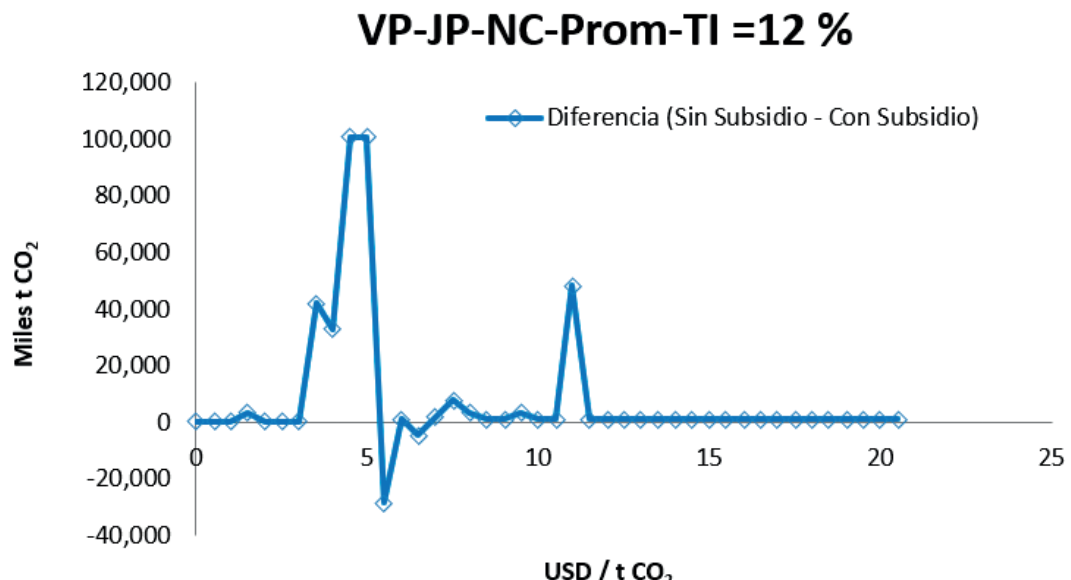

Figura 11. Efecto del subsidio en relación con el potencial de reducción de emisiones (superficies en riesgo de deforestación/ degradación). 
En la figura 12 se observa que con costo de oportunidad de USD 1.5/t $\mathrm{CO}_{2}{ }^{-1}$, el potencial de reducción de emisiones es de 200000000 t $\mathrm{CO}_{2}$ para los casos de solo considerar actividades de costo mínimo. Este potencial de reducción tiene un costo de USD 5.5/t $\mathrm{CO}_{2}^{-1}$ para actividades con costo promedio y USD $19 / \mathrm{CO}_{2}^{-1}$ para actividades con costos máximos.

La figura 13 muestra la situación de costos de oportunidad positivos promedio, sin pérdida de empleo, sin crédito y con subsidio, para el efecto de la TI.
En la figura 13 se observa que para un potencial de reducción de emisiones de 200000000 t $\mathrm{CO}_{2}$, el costo es de USD 4.5/t $\mathrm{CO}_{2}^{-1}$ para una TI de $6 \%$, de USD 5.5/t CO ${ }_{2}^{-1}$ para una TI de $12 \%$ y USD $10.5 / \mathrm{t} \mathrm{CO}_{2}^{-1}$ para una de $18 \%$, mostrando el efecto del valor del dinero en el tiempo.

La figura 14 muestra el efecto del crédito en los escenarios de mitigación para el caso de costos de oportunidad promedio positivos, con subsidio y $\mathrm{TI}=12 \%$.

La figura 15 permite visualizar en forma más adecuada el efecto del crédito en los potenciales de mitigación

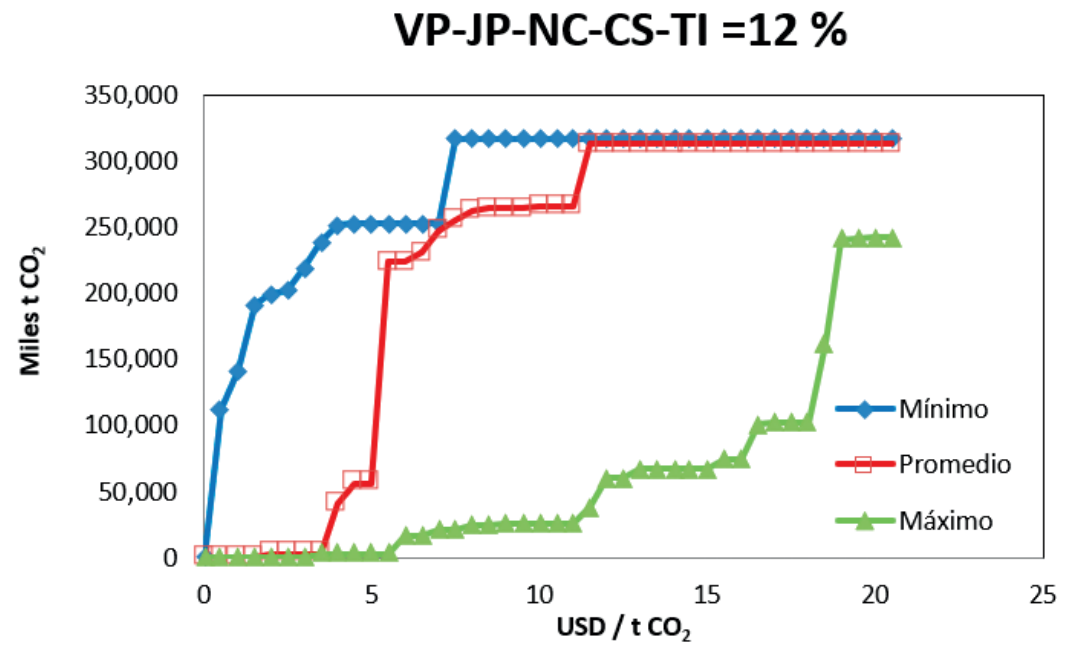

Figura 12. Efecto del valor del costo de oportunidad para el escenario de mitigación: VP: costos de oportunidad positivos, JP: sin pérdida de empleo, NC: no crédito, CS: con subsidio y TI (tasa de interés) = 12\% (superficies en riesgo de deforestación/degradación).

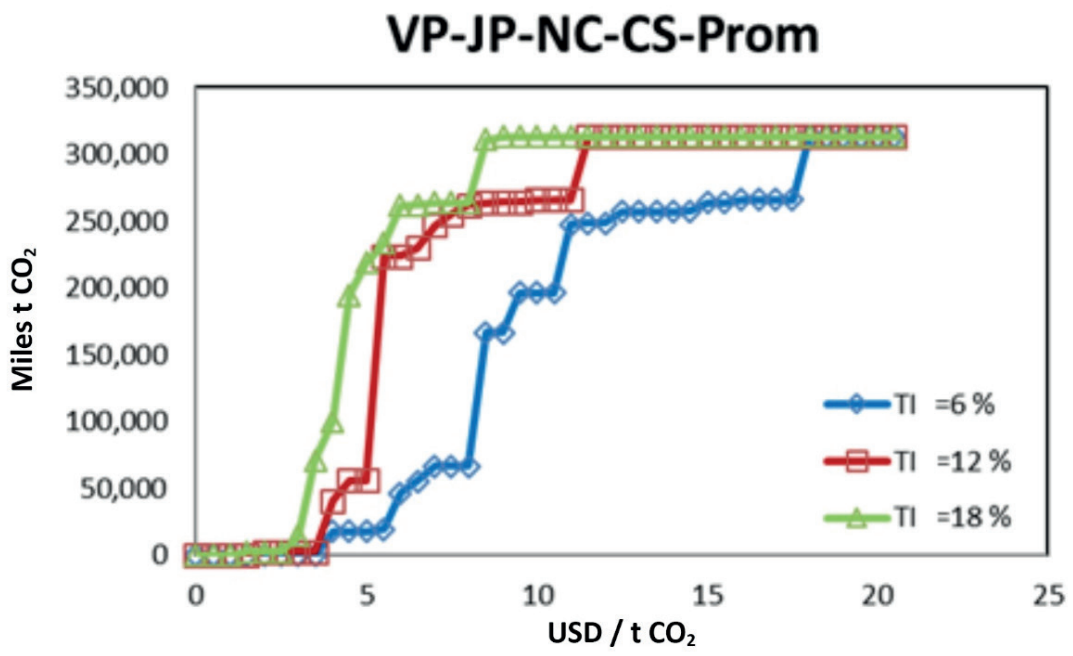

Figura 13. Efecto de la TI (tasa de interés) para el escenario de mitigación: VP: costos de oportunidad positivos, JP: sin pérdida de empleo, NC: sin crédito, CS: con subsidio y Prom: costos de oportunidad promedio (superficies en riesgo de deforestación/degradación). 


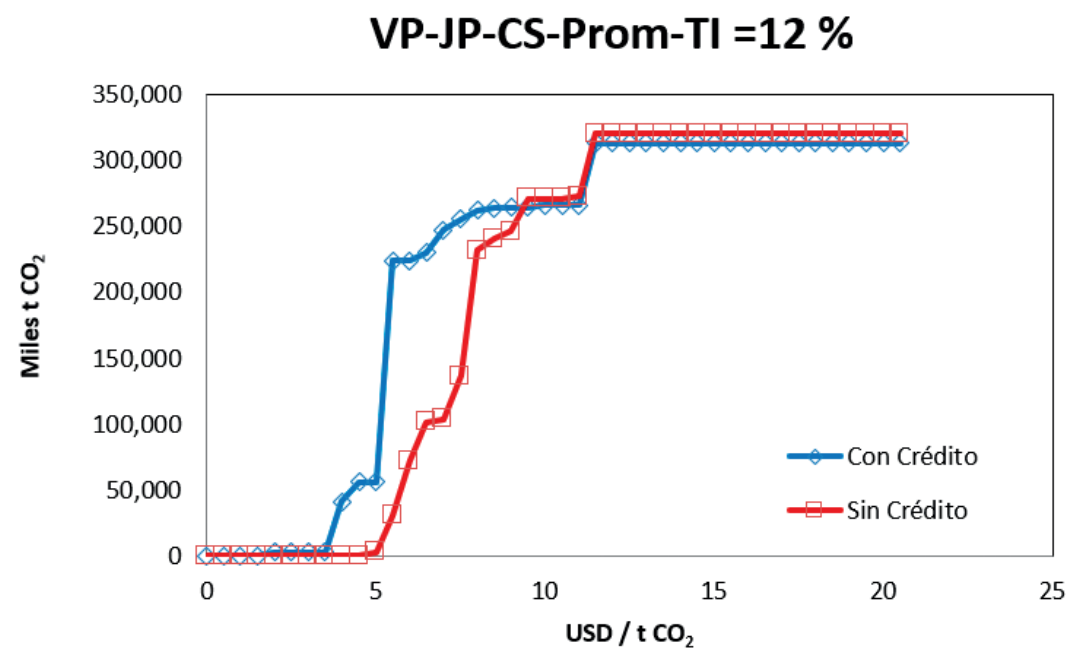

Figura 14. Efecto del crédito para el escenario de mitigación: VP: costos de oportunidad positivos, JP: sin pérdida de empleo, CS: con subsidio, Prom: costos de oportunidad promedio y TI (tasa de interés) = 12\% (superficies en riesgo de deforestación/degradación).

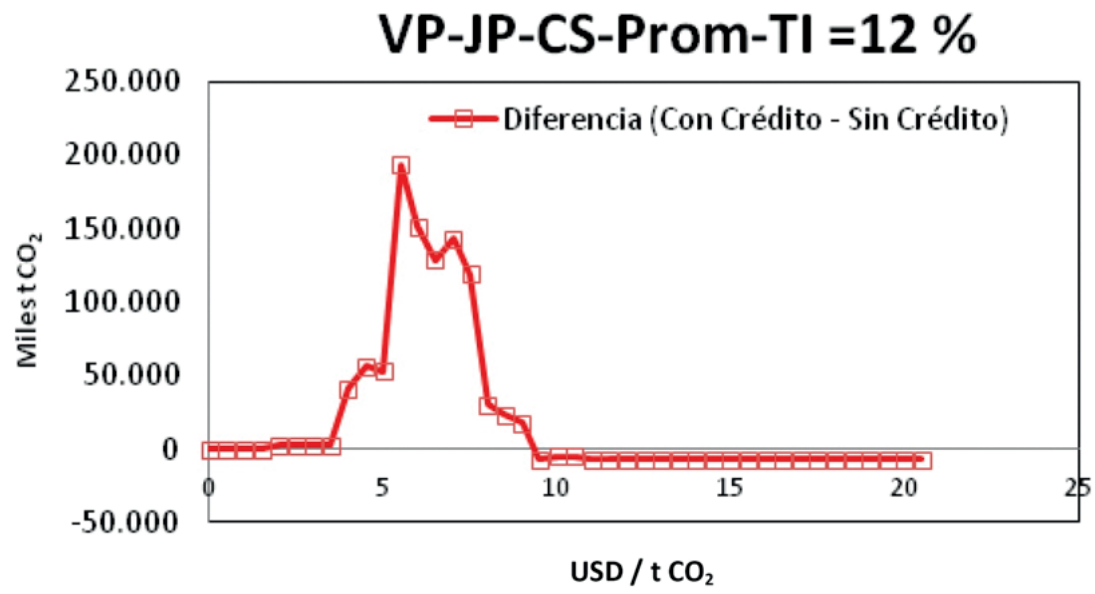

FigURA 15. Efecto del crédito en relación con el potencial de reducción de emisiones (superficies en riesgo de deforestación/degradación).

y sus costos de oportunidad asociados. Esta figura muestra que para costos de oportunidad entre USD 3.5/t $\mathrm{CO}_{2}^{-1} \mathrm{y}$ USD 5.0/t $\mathrm{CO}_{2}^{-1}$, el no utilizar crédito implica una pérdida de potencial de reducción de alrededor de $200000000 \mathrm{t}$ $\mathrm{CO}_{2}$. Para valores mayores a USD 10.0/t $\mathrm{CO}_{2}^{-1}$, este efecto negativo se reduce hasta ser prácticamente nulo.

\section{Escenarios de mitigación con costos de oportunidad positivos y componentes de REDD+}

La figura 16 muestra los potenciales de reducción de emisiones de REDD+ (todos los componentes), así como de solo considerar la deforestación o la degradación forestal.
La figura 17 muestra el diferencial de reducción de emisiones de GEI de solo considerar la deforestación en relación con todos los componentes de REDD+, donde se observa que los diferenciales son cercanos a cero, pero negativos y, solo para el caso de USD 5.0/t $\mathrm{CO}_{2}^{-1}$ (actividades de degradación, principalmente), la situación de considerar un componente adicional a la deforestación conlleva una pérdida del potencial de reducción de emisiones.

La figura 18 muestra el diferencial de reducción de emisiones de GEI de solo considerar la degradación forestal en relación con todos los componentes de REDD+, donde se 


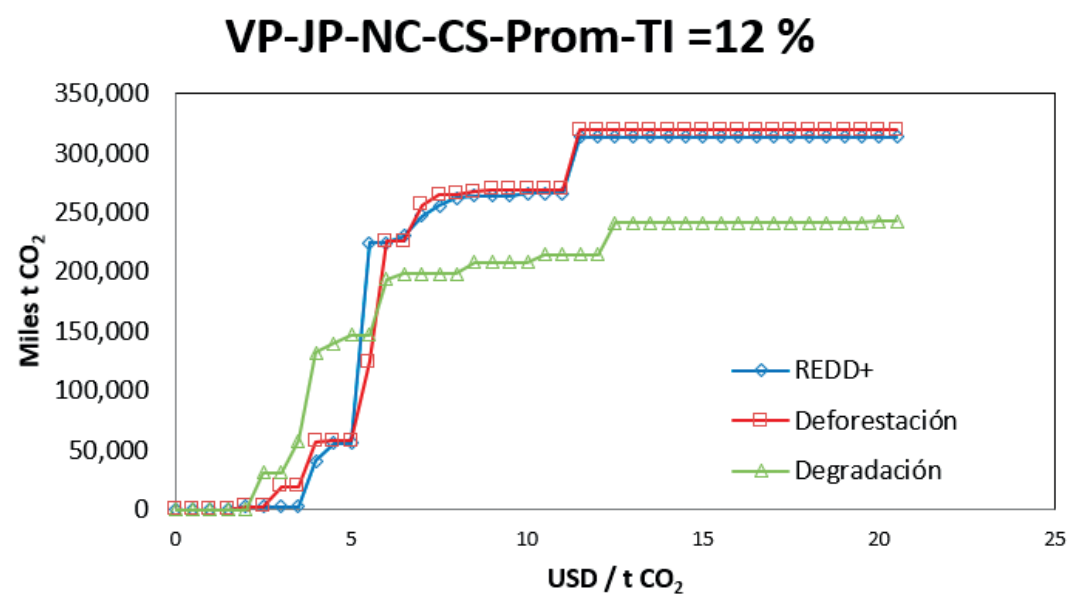

Figura 16. Efecto de las componentes de REDD+ para el escenario de mitigación: VP: costos de oportunidad positivos, JP: sin pérdida de empleo, NC: sin crédito, CS: con subsidio, Prom: costos de oportunidad promedio, TI (tasa de interés) = 12\%; para REDD+ (todos sus componentes), deforestación y degradación forestal (superficies en riesgo de deforestación/degradación).

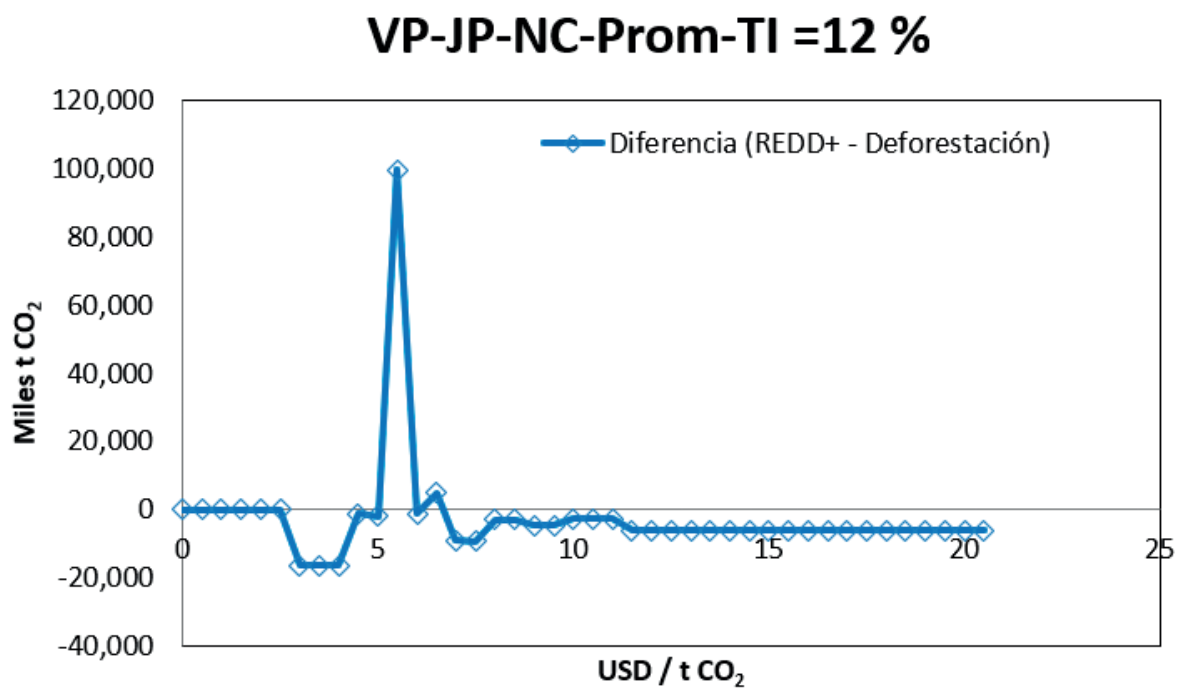

Figura 17. Efecto de considerar solo deforestación en relación con REDD+ (todos los componentes) en relación con el potencial de reducción de emisiones (superficies en riesgo de deforestación/degradación).

observa que los diferenciales son importantes (pérdidas del potencial) en el intervalo de USD 2.0/t $\mathrm{CO}_{2}^{-1}$ a USD 5.0/t $\mathrm{CO}_{2}^{-1}$. Por encima de USD 5.0/t $\mathrm{CO}_{2}^{-1}$, estos efectos son revertidos.

Finalmente, la figura 19 muestra el potencial de la degradación forestal en relación con considerar solo la deforestación, donde se observa un patrón similar al caso de la diferencia entre REDD+ (todos los componentes) y la deforestación.

\section{Oportunidades de mitigación con costos de} oportunidad negativos

La tabla 6 muestra el potencial de mitigación de una reorientación de políticas públicas que incentiven cambios de actividades con costos de oportunidad negativos (en el umbral de costo cero) en áreas boscosas en riesgo de deforestación/degradación forestal, considerando el efecto de diversas restricciones (sin restricciones; sin pérdida de empleo y con crédito; y sin pérdida de empleo y sin crédito). 


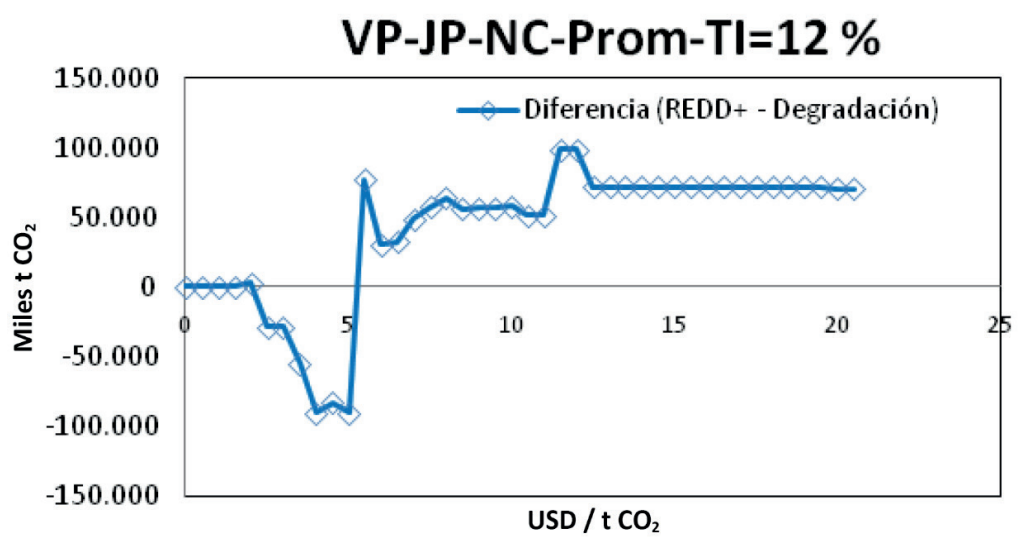

FIgURA 18. Efecto de considerar solo la degradación forestal en relación con REDD+ (todos los componentes) en relación con la reducción del potencial de emisiones (superficies en riesgo de deforestación/degradación).

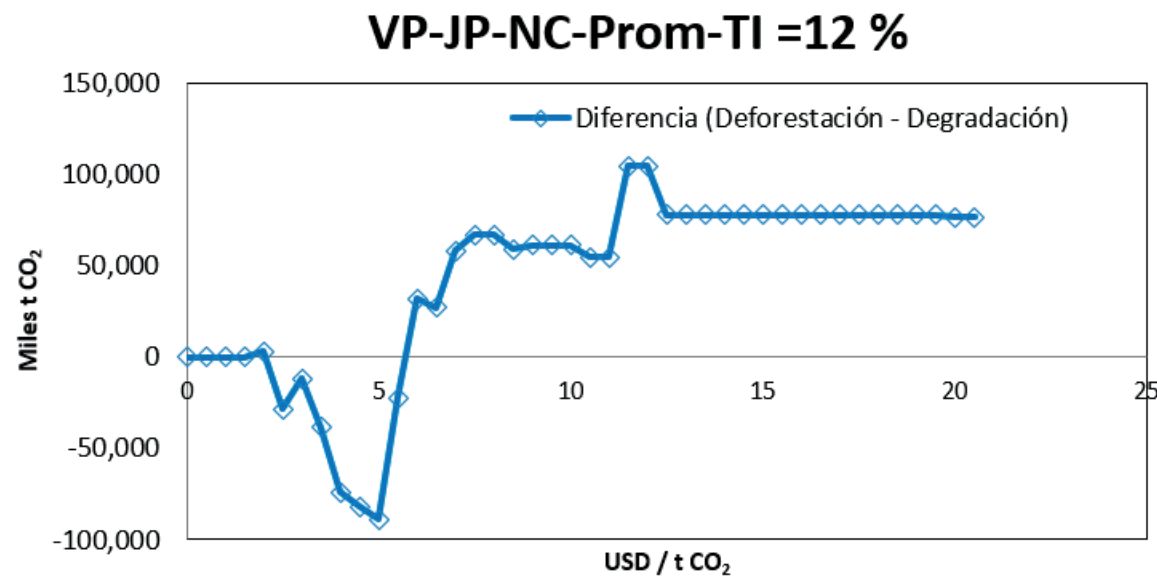

Figura 19. Efecto de considerar solo degradación forestal en relación con solo deforestación, sobre el potencial de reducción de emisiones (superficies en riesgo de deforestación/degradación).

\section{Potenciales de mitigación regionalizados para la} focalización de acciones REDD+

Para analizar los potenciales de mitigación regionales, la tabla 7 muestra los resultados obtenidos para las ocho regiones en que fue dividido Chiapas (Fig. 2), tanto para costos de oportunidad promedios negativos como para costos de oportunidad promedio positivos, considerando en ambos casos que no hay pérdidas de empleo ni crédito.

El análisis regional efectuado señala que existen diferencias en cuanto a potenciales de mitigación y costos para las distintas regiones de Chiapas. Las regiones Altos y Sierra Madre son las que presentan un mayor potencial de mitigación para costos de oportunidad negativos. En el caso de costos de oportunidad positivos, las regiones Selva Maya y Altos son las que presentan un mayor potencial de mitigación.

\section{DISCUSIÓN}

\section{Curvas de costos de abatimiento unitarias y factores asociados}

Las curvas de costos de abatimiento muestran el costo de emprender distintas acciones de mitigación de emisiones, permitiendo ver cuáles son las más costo-efectivas. El análisis de las curvas generadas para el estado de Chiapas permite observar que existen actividades con diferentes 
Tabla 6. Potencial de mitigación a no costo.

\begin{tabular}{ccc}
\hline Escenario & $\begin{array}{c}\text { Estadístico del Costo de } \\
\text { Oportunidad }\end{array}$ & $\mathrm{tCO}_{2}$ (miles) \\
\hline CS_VN & Min & 081890.88 \\
& Prom & 132945.40 \\
CS_VN-JP-SC & Max & 158190.40 \\
& Min & 081117.91 \\
CS_VN-JP-NC & Prom & 087743.89 \\
& Max & 102267.30 \\
& Min & 029071.01 \\
& Prom & 058867.93 \\
& Max & 091122.98 \\
\hline
\end{tabular}

CS_VN: con subsidio, costos de oportunidad negativos (sin restricciones); CS_ VN-JP-SC: con subsidio, costos de oportunidad negativos, sin pérdida de empleo y con crédito; CS_VN-JP-NC: con subsidio, costos de oportunidad negativos, sin pérdida de empleo y sin crédito.

potenciales de mitigación y a diferentes costos, lo que facilita la clasificación y selección de actividades de implementación. De esta manera, los tomadores de decisiones pueden elegir, por ejemplo, un intervalo de costos de oportunidad de acuerdo con presupuestos y objetivos políticos (e.g., < USD 10/t $\mathrm{CO}_{2}$ ) y acotar el elenco de medidas a aplicar. A su vez, es posible evaluar la costo-eficiencia de una medida concreta que sea de especial interés para los objetivos políticos estatales o regionales (e.g., incentivar el manejo sostenible de acahuales) o identificar las medidas con mayor potencial de mitigación y sus costos asociados.

En relación con REDD+, resulta claro que incluye componentes con costos de oportunidad similares pero potenciales de reducción de emisiones diferentes. Tal es el caso de la deforestación, que presenta un mayor potencial de mitigación que la degradación. En este sentido, se resalta que evitar la deforestación de los ecosistemas forestales que albergan las mayores cantidades de carbono en el estado -esto es, selvas altas perennifolias, bosques mesófilos y manglares- presenta los mayores potenciales de mitigación con bajos costos de oportunidad, aunque varían en función del uso final del terreno. Estos resultados coinciden con los mostrados por la curva de costos de abatimiento de GEI para México en 2020, en la que la reducción de la deforestación por conversión a pastizales y por quema y tala en la agricultura presentan bajos costos de abatimiento.

La degradación forestal ostenta, en principio, un menor potencial de mitigación que la deforestación, aunque en muchas ocasiones, ambas dinámicas van unidas: primero se extraen los productos de valor (madera o leña) $y$, una vez agotados, el bosque deja de aportar ingresos por lo que puede optarse por la tala para usos agrícolas o pecuarios (Covaleda et al., 2014). Adicionalmente, algu-

Tabla 7. Potencial de mitigación por regiones de Chiapas para distintos costos de oportunidad.

\begin{tabular}{|c|c|c|c|}
\hline Región & $\begin{array}{l}\text { Costo de oportunidad negativo } \\
\qquad\left(\text { miles } t \mathrm{CO}_{2}\right)\end{array}$ & $\begin{array}{c}\text { USD } 5 \text { t } \mathrm{CO}_{2}-\mathrm{USD} 6 / \mathrm{tCO}_{2} \\
\left(\text { miles } \mathrm{C} \mathrm{CO}_{2}\right)\end{array}$ & $\begin{array}{c}\text { USD } 6 / \mathrm{t} \mathrm{CO}_{2}-\mathrm{USD} 7 / \mathrm{tCO} \mathrm{CO}_{2} \\
\left(\text { miles } \mathrm{C} \mathrm{CO}_{2}\right)\end{array}$ \\
\hline Selva Maya & 02914 & 43924 & 65990 \\
\hline Selva Zoque & 05028 & 15069 & 15069 \\
\hline Soconusco & 01288 & 05405 & 05405 \\
\hline Llanura Costera & 02657 & 07579 & 07579 \\
\hline Depresión Central & 00000 & 18570 & 18579 \\
\hline Norte & 01512 & 03988 & 04128 \\
\hline Altos & 06383 & 30130 & 41721 \\
\hline Sierra Madre & 06473 & 11390 & 23539 \\
\hline
\end{tabular}


nas actividades relacionadas con la degradación forestal son altamente rentables, como la extracción de determinadas especies arbóreas para la venta de madera o la producción de café (en algunos casos), por lo que sus costos de oportunidad son elevados. Por ello, se deben buscar estrategias adecuadas para cada caso: buscar la recuperación de los reservorios de carbono forestal o evitar ulteriores pérdidas (deforestación).

Además del potencial de mitigación y sus costos, cada actividad que pretenda ponerse en marcha (estado final) presenta unos requerimientos laborales específicos (jornales) que no tienen por qué ser similares a los del estado inicial y que es importante tener en cuenta. Esto se debe a que un mayor requerimiento laboral de la actividad final puede ser positivo, en caso de que favorezca la creación de empleo a escala local; o negativo si el productor tiene que cubrir el incremento de jornales con mano de obra familiar, restando tiempo a otras labores, lo cual puede ser un impedimento importante si este mayor esfuerzo no es compensado con un mayor beneficio económico.

\section{Escenarios de mitigación de emisiones}

La generación de escenarios de mitigación asociados con acciones REDD+ y acciones de mitigación en el sector agropecuario con los METs permite valorar los requerimientos financieros de la aplicación operacional de distintas medidas, además de generar información que permita la cuantificación de los efectos de diversas políticas públicas y decisiones de inversión en la reducción de emisiones de GEI. Adicionalmente, asociados con los escenarios de mitigación, es posible analizar los efectos colaterales (riesgos) de la implementación de REDD+, tales como la pérdida de empleo o requerimientos de crédito.

Los METs regionales elaborados en Chiapas se construyeron con estados identificados a escala local, de manera que es posible conocer el impacto que supondría la transformación de diferentes tipos de bosques y selvas en otros usos del suelo comunes en una determinada región, permitiendo conocer las pérdidas de carbono en cada caso y los costos de oportunidad asociados, de tal manera que es posible estimar los costos de mantener los bosques en pie en función de los usos productivos con los que compiten. La consideración de la biodiversidad y funciones ecológicas, sin embargo, no está incluida en la presente aproximación.

Un problema de la aproximación utilizada en Chiapas para la construcción de los modelos es que se consideraron las denominaciones locales de los usos del suelo, lo que dificulta la homologación de las clases utilizadas por otros sistemas de clasificación de usos del suelo empleados en el país como los utilizados por el Inegi o el Sistema de Información Agroalimentaria y Pesquera [SIAP] de la Sagarpa.

Normalmente, tras el cambio de uso del suelo, los sistemas de manejo que se establecen utilizan técnicas convencionales (Gudynas y Ghionas, 2010), por lo que, si se pretende aumentar los almacenes de carbono en sistemas agrícolas y pecuarios, los METs permiten plantear escenarios para conocer el impacto del establecimiento de prácticas sostenibles de tal manera que puedan seleccionarse las prácticas más adecuadas en función de los objetivos. Por tanto, los METs facilitan el planteamiento de escenarios de mitigación "a medida”, de manera que en un proceso de planificación municipal, regional o estatal, en el que se busca alcanzar metas de mitigación específicas, estos modelos pueden ayudar a seleccionar las actividades más adecuadas y a conocer los costos asociados, así como el impacto sobre estos costos y potenciales de mitigación de diferentes restricciones (tasa de interés, subsidio, empleo, crédito). Los análisis pueden realizarse seleccionando previamente una actividad o un número limitado de actividades para un municipio, región o para todo el estado o tomando todo el elenco de actividades disponibles y estableciendo distintos tipos de restricciones, con el fin de tener una visión global de las opciones existentes.

En relación con el análisis realizado se obtuvo que, en Chiapas, para alcanzar un potencial de mitigación de 200 000000 de $\mathrm{t} \mathrm{CO}_{2}$ pueden seleccionarse actividades con costos mínimos (USD 1/t $\mathrm{CO}_{2}^{-1}$ ), promedios (USD 7.5/t $\mathrm{CO}_{2}^{-1}$ ) o máximos (USD 19/t $\mathrm{CO}_{2}^{-1}$ ). En caso de que se consideren únicamente actividades que no supongan pér- 
dida de empleo, el costo promedio para este potencial de reducción sería de USD 5.5/t $\mathrm{CO}_{2}^{-1}$.

En cuanto a los efectos de las restricciones, el subsidio tuvo un efecto negativo para costos de oportunidad entre USD 2.5/t $\mathrm{CO}_{2}^{-1}$ y USD 5.0/t $\mathrm{CO}_{2}^{-1}$, al igual que el no contar con crédito para costos de oportunidad entre USD 3.5/t $\mathrm{CO}_{2}^{-1}$ y USD 5.0/t $\mathrm{CO}_{2}^{-1}$. Tener en cuenta estas consideraciones asegura una planificación económica más efectiva de las acciones de implementación y permite contar con elementos clave para el diseño de políticas públicas y financieras concretas.

Otra utilidad de los modelos es que permiten comparar el potencial de reducción de diferentes tipos de actividades como, por ejemplo, los diferentes componentes de REDD+. Los resultados obtenidos señalan que los esfuerzos encaminados a reducir la deforestación permiten alcanzar una mayor reducción de emisiones; sin embargo, hay que tener en cuenta que las actividades asociadas a procesos de deforestación son las que predominan en los análisis de los modelos de estados y transiciones, así como en los costos de oportunidad. Es necesario, por tanto, contar con un mayor registro de actividades de degradación para poder hacer comparaciones más detalladas. Lo mismo ocurre con el manejo forestal sostenible (para producción maderable) y los incrementos en los almacenes de carbono, tanto forestales como agropecuarios. Esta carencia es debida a que el número de sistemas alternativos o sostenibles a evaluar está restringido a la disponibilidad de información sobre los mismos en relación con todas las variables que se incluyen en los modelos, lo que ha limitado la inclusión de algunos sistemas o variantes de manejo. Es necesario, por tanto, dedicar mayores esfuerzos de investigación a estos temas.

Por último, las actividades que presentan costos de oportunidad negativos permiten tener una idea de las acciones de gobierno que pueden ser logradas en forma relativamente simple (reorientación de programas de apoyo, federales y estatales, a productores) y que pueden ser el escenario de referencia de acreditación en Chiapas, considerando las superficies boscosas en riesgo de deforestación/degradación forestal.

\section{CONCLUSIONES}

En el marco actual de preparación e aplicación de los nuevos mecanismos de mitigación de emisiones en el sector Afolu en la Cmnucc, es fundamental contar con herramientas como los modelos de estados y transiciones para planear acciones y políticas públicas efectivas. Estos modelos permiten, además, valorar el impacto de la puesta en marcha de diferentes actividades en términos económicos, de empleo, necesidades de subsidio y crédito.

Los METs elaborados en Chiapas cuentan con un número restringido de actividades que son reflejo de la información disponible, centrada principalmente en las dinámicas de deforestación. Por ello y para mejorar el potencial de análisis de esta herramienta, es necesario incentivar la investigación y generar conocimiento en relación con las actividades asociadas a la degradación forestal, manejo forestal sostenible e incremento de almacenes de carbono forestal en el caso de REDD+ y más actividades asociadas al desarrollo rural sostenible de bajas emisiones. La homologación de las clases de uso del suelo y vegetación utilizadas en los METs con las empleadas por otras instituciones mexicanas requiere ser afinada en las nuevas versiones de los modelos de estados y transiciones, para mejorar la calidad de los análisis y facilitar la incorporación de nueva información a los modelos.

Del análisis efectuado en Chiapas utilizando estos modelos, se concluye que el estado presenta un gran potencial de mitigación de emisiones en el sector Afolu, en particular las regiones Selva Maya y Altos, por lo que las acciones encaminadas a la implementación del mecanismo REDD+ en Chiapas deberían considerar, al menos, estas regiones como prioritarias.

En lo general, del análisis de los diferentes escenarios revisados, un costo de oportunidad de USD 4/t $\mathrm{CO}_{2}$ a USD 6/t $\mathrm{CO}_{2}$ parece ser suficiente para una buena porción del potencial de mitigación de GEI de Chiapas, aunque en forma de óptimos (costos mínimos) generales, con USD 1/t $\mathrm{CO}_{2}^{-1}$ - USD 2/t $\mathrm{CO}_{2}^{-1}$ sería suficiente. 


\section{RECONOCIMIENTOS}

Los autores agradecen a Ma. Isabel Marín el apoyo brindado en la homologación de clases de los METs con las series del mapa de Inegi y al Programa Mexicano del Carbono y Conservation International México A. C. por el financiamiento para el desarrollo de los modelos y su contribución a la difusión de estos trabajos.

\section{REFERENCIAS}

Ambio. (2012). The Scolel Te Program, 2011 Annual Report. Recuperado de http://www.planvivo.org/project-network/ scolelte-mexico/

Australian Government. (2011). Estimating the cost of abatement. Framework and practical guidance. Barton: Department of Climate change and Energy efficiency.

Castillo, M. A., de Jong, B., Maldonado, V., Rojas, F., Olguín, M., de la Cruz, V., Paz, F., \& Jiménez, G. (2010). Modelo de deforestación para el estado de Chiapas. Recuperado de http://www.pmcarbono.org/pmc/biblioteca/doc_socios. php.

Clerc, J., Díaz, M., \& Campos, B. (2013). Desarrollo de una metodología para la construcción de curvas de abatimiento de emisiones de GEI incorporando la incertidumbre asociada a las principales variables de mitigación. Banco Interamericano de Desarrollo, Nota técnica 541. Recuperado de https://publications.iadb.org/handle/113 19/5994? locale-attribute $=$ es \&scope $=123456789 / 11 \&$ thu mbnail=false\&order=desc\&rpp=5\&sort_by=score \&page $=1 \&$ query=Informe+Mercosur+18\&group_by=none\&etal $=0 \&$ filtertype $0=$ subject_en $\&$ filter_ $0=$ Greenhouse + gases \&filter_relational_operator_0=equals.

Comisión Nacional para el Conocimiento y Uso de la Biodiversidad [Conabio]. (2013). Estrategia para la conservación y el uso sustentable de la biodiversidad del estado de Chiapas.

México D. F. Comisión Nacional para el Conocimiento y Uso de la Biodiversidad.

Comisión Nacional Forestal [Conafor]. (2016). Documento de la Iniciativa de Reducción de Emisiones. Recuperado de http://www.conafor.gob.mx:8080/documentos/ docs/35/6908Borrador $\% 20$ de $\% 201 \mathrm{a} \%$ 20Iniciativa $\% 20$ de\%20Reducci\%C3\%B3n\%20de\%20Emisiones.pdf .
Convención Marco de Naciones Unidas sobre el Cambio Climático [Cmnucc]. (2015). The Paris Agreement. Recuperado de http://unfccc.int/paris_agreement/items/9485.php

Covaleda, S. (2010). Modelos de estados y transiciones para los almacenes de carbono en las principales regiones de Chiapas. (Reporte de estancia postdoctoral). El Colegio de la Frontera Sur y el Colegio de Postgraduados. San Cristóbal de las Casas.

Covaleda, S., Paz, F., \& de Jong, B. (2012a). Modelo genérico de estados y transiciones para los cambios en los almacenes de carbono en ecosistemas templados de Chiapas. En F. Paz, \& R. Cuevas (Eds.), Estado Actual del Conocimiento del Ciclo del Carbono y sus Interacciones en México: Síntesis a 2011. Serie Sintesis Nacionales (pp. 356-363). Texcoco: Programa Mexicano del Carbono - Universidad Autónoma del Estado de México - Instituto Nacional de Ecología.

Covaleda, S., Paz, F., \& de Jong, B. (2012b). Modelos de estados y transiciones: una herramienta para la planificación de estrategias REDD+. En F. Paz, \& R. Cuevas Eds.), Estado Actual del Conocimiento del Ciclo del Carbono y sus Interacciones en México: Sintesis a 2011. Serie Sintesis Nacionales (pp.773-739). Texcoco: Programa Mexicano del Carbono - Universidad Autónoma del Estado de México Instituto Nacional de Ecología.

Covaleda, C., Paz, F., \& de Jong, B. (2013). Parametrización de modelos de estados y transiciones para el carbono y caracterización de la incertidumbre. En. F. Paz, M. Bazan, \& V. Saynes (Eds.), Dinámica del Carbono en el Suelo 2012. Serie Avances Temáticos del Ciclo del Carbono y sus Interacciones (pp. 85-91). Texcoco: Programa Mexicano del Carbono - la Sociedad Mexicana de Ciencias del Suelo.

Covaleda, S. (2014). Actualización de los modelos de estados y transiciones de las regiones Sierra Madre, Soconusco, Llanura Costera y Depresión Central. Kibeltik Clima y Medio Ambiente A. C. Tuxtla-Gutiérrez: Conservación Internacional México A. C.

Covaleda, S., Ranero, A., \& Aguilar, S. (2014). Diagnóstico de determinantes de deforestación en Chiapas. Recuperado de http://www.pmcarbono.org/pmc/biblioteca/doc_socios.php

Covaleda, S., Paz, F., \& Ranero, A. (2016). Carbono edáfico en Chiapas: Planteamiento de políticas públicas de mitigación de emisiones. Terra Latinoamericana, 34(1), 97-112. 
De Jong, B., Rojas, F., Olguín, M., de la Cruz, V., Paz, F., Jiménez, G., \& Castillo, M. A. (2010). Establecimiento de una linea base de las emisiones actuales y futuras de Gases de Efecto Invernadero provenientes de Agricultura, Silvicultura y otros usos del suelo. Recuperado de http://www. pmcarbono.org/pmc/biblioteca/doc_socios.php.

Eagle, A. J., Henry, L. R, Olander, L. P., Haugen-Kozyra, K., Millar, N., \& Robertson G. P. (2011). Greenhouse gas mitigation potential of agricultural land management in the United States, a synthesis of the literature. Technical Working Group on Agricultural Greenhouse Gases (T-AGG) Report (2 ${ }^{\text {nd }}$ ed). Durham: Nicholas Institute for Environmental Policy Solutions.

Earth Innovation Institute (2015). Promovendo o desenvolvimento rural de baixas emissões. Recuperado de http://earthinnovation.org/wp-content/uploads/2015/01/ LED-R-report_pt_2015.pdf.

Gudynas, E., \& Ghionas, S. (2010). Agricultura y ganadería, biodiversidad, cambio climático: Estrechamente vinculados. LEISA Revista de Agroecología, 26, 40-43.

Hansen, M. C., Potapov, P., Moore, V., Hancher, R., Turubanova, M., Tyukavina, S. A., Thau, A. D., Stehman, S. V., Goetz, S. J., Loveland, T. R., Kommareddy, A., Egorov, A., Chini, L., Justice C. O., \& Townshend, J. R. G. (2013). High-resolution global maps of 21st-century forest cover change. Science, 342, 850-853. doi: 10.1126/science.1244693

Instituto Nacional de Estadística, Geografía e Informática Comisión Nacional para el Conocimiento y Uso de la Biodiversidad - Instituto Nacional de Ecología [Inegi -Conabio-INE]. (2009). México: Ecorregiones terrestres de México. Escala 1:1000 000.

Jiménez, G., Marinidou, E., González, A., de Jong, B., Ochoa, S., \& Olguín, M. (2010). Establecimiento de una línea base de las emisiones actuales y futuras de Gases de Efecto Invernadero provenientes de los subsectores Agricultura $y$ Ganadería, del sector Agricultura, Silvicultura y otros usos del suelo (Afolu 1.2). Tuxtla Gutiérrez: Conservación Internacional México A. C.

Mullerried, F. K. G. (1957). La geología de Chiapas. Colección Libros de Chiapas, Serie Básica. Tuxtla-Gutiérrez: Gobierno del Estado de Chiapas.
Paz, F., Marín, M. I., Medrano, E. R, Ibarra F., \& Pascual, F. (2010). Elaboración de mapas multi-temporales de bosque, a partir de imágenes LANDSAT, TM y ETM+ y análisis de la degradación forestal y deforestación en Chiapas. Recuperado de http://www.pmcarbono.org/pmc/biblioteca/doc_socios.php.

Paz, F., Covaleda, S., Ranero, A., Ugarte, X., Esquivel, E., Marín, M. I., Cuevas, R., de Jong, B., \& Etchevers, J. D. (2012). Estudio de Factibilidad para el mecanismo REDD+ en Chiapas. Recuperado de http://www.pmcarbono.org/pmc/ proyectos/CI_Factibilidad_REDD+.php.

Paz, F. (2015). ¿Es suficiente evaluar "datos de actividad x factores de emisión = emisiones" en mecanismos tipo REDD+o RETUS. En F. Paz, \& J. Wong (Eds.), Estado Actual del Conocimiento del Ciclo del Carbono y sus Interacciones en México: Síntesis a 2014. Serie Sintesis Nacionales (pp. 526-532). Mérida: Programa Mexicano del Carbono Centro de Investigaciones y Estudios Avanzados del Instituto Politécnico Nacional - Unidad Mérida - Centro de Investigación y Asistencia en Tecnología y Diseño del Estado de Jalisco.

Paz, F., \& Covaleda, S. (2015). Modelos de estados y transiciones (METs) compuestos para la modelación anual de la dinámica del carbono. En F. Paz, \& J. Wong (Eds.), Estado Actual del Conocimiento del Ciclo del Carbono y sus Interacciones en México: Síntesis a 2014. Serie Sintesis Nacionales (pp. 541-546). Mérida: Programa Mexicano del Carbono - Centro de Investigaciones y Estudios Avanzados del Instituto Politécnico Nacional - Centro de Investigación y Asistencia en Tecnología y Diseño del Estado de Jalisco.

Paz, F., Covaleda, S., \& de Jong, B. (2015). Modelos de la dinámica temporal del carbono orgánico de los suelos asociada a cambios de uso del suelo en ecosistemas forestales. En F. Paz, \& J. Wong (Eds.), Estado Actual del Conocimiento del Ciclo del Carbono y sus Interacciones en México: Síntesis a 2014. Serie Síntesis Nacionales (pp. 363-368). Mérida: Programa Mexicano del Carbono - Centro de Investigaciones y Estudios Avanzados del Instituto Politécnico Nacional - Centro de Investigación y Asistencia en Tecnología y Diseño del Estado de Jalisco. 
Reyes, M., Covaleda, S., Perez, M. J., \& Paz, F. (2012). Ordenamientos territoriales e intervenciones a escala local en Chiapas en REDD+: carbono, costos de oportunidad y modelos de estados y transiciones. En F. Paz, \& R. Cuevas (Eds.) Estado Actual del Conocimiento del Ciclo del Carbono y sus Interacciones en México: Síntesis a 2011. Serie Sintesis Nacionales (pp. 680-684). Texcoco: Programa Mexicano del Carbono - Universidad Autónoma del Estado de México - Instituto Nacional de Ecología.

Secretaría de Medio Ambiente e Historia Natural del Estado de Chiapas [Semahn]. (2017). Documento de la Estrategia Estatal REDD+ del Estado de Chiapas. Recuperado de http://www.semahn.chiapas.gob.mx/portal/descargas/ cambio_climatico/eeredd_130317.pdf

Secretaría de Medio Ambiente e Historia Natural del Estado de Chiapas [Semahn]. (2011). Programa de Acción Ante el Cambio Climático del Estado de Chiapas. Recuperado de http:/www.cambioclimaticochiapas. org/portal/descargas/ pacch/paccch.pdf

Stringham, T. K., Krueger, W. C., \& Shaver, P. L. (2001). States, transitions and thresholds: further refinement for rangeland applications, Special Report 1024. Corvallis: Agricultural Experiment Station, Oregon State University.
Vaca, R. A., Golicher, D. J., Cayuela, L., Hewson, J., \& Steininger, M. (2012). Evidence of incipient forest transition in Southern Mexico. PLoS ONE, 7(8), e42309. doi:10.1371/ journal.pone.0042309.

Westoby, M., Walker, B., \& Noy-Meir, I. (1989). Opportunistic management for rangelands not at equilibrium. Journal of Rangeland Management, 42, 266-274.

White, D., \& Minang, P. (2011). Estimación de los costos de oportunidad de REDD+ Manual de capacitación. Versión 1.3. Washington D. C. Banco Mundial.

Yin, X., Gourdriaan, J., Lantinga, E. A., Vos, J., \& Spiertz, H. J. (2003). A flexible sigmoid function of determinate growth. Annals of Botany, 91, 361-371.

Manuscrito recibido el 13 de julio de 2017.

Aceptado el 21 de enero de 2018.

Publicado el 17 de diciembre de 2018.

Este documento se debe citar como:

Covaleda, S., Paz-Pellat, F. \& Ranero, A. (2018). Escenarios de mitigación de emiciones para el sector rural del estado de Chiapas utilizando modelos de estados y transiciones. Madera y Bosques, 24(Núm. esp.), e2401897. doi: 10.21829/myb.2018.2401897

Madera y Bosques por Instituto de Ecología, A.C. se distribuye bajo una Licencia Creative Commons Atribución-No Comercial-Compartir Igual 4.0 Internacional. 